\title{
Transición de la escuela al trabajo. Tres décadas de evidencia para América Latina
}

\author{
Mariana Viollaz
}

RESUMEN

En este trabajo se analiza la inserción de los jóvenes en el mercado laboral en diez países de América Latina durante las tres últimas décadas utilizando encuestas de hogares. Se constata que: i) la situación de los jóvenes se deterioró en el tiempo con una mejora hacia finales de la década de 2000. Pese a ello, las tasas de desempleo e informalidad juvenil se mantienen en niveles muy elevados; ii) los jóvenes logran ingresar en una trayectoria típica del ciclo de vida laboral, superando los resultados obtenidos por adultos de generaciones anteriores. La informalidad laboral no se ajusta a este comportamiento e indica la existencia de algún tipo de penalidad vinculada a la condición de informalidad en la juventud. En términos generales, los resultados son alentadores. Se concluye que los esfuerzos realizados para mejorar la situación de los jóvenes en el mercado de trabajo deben mantenerse para prolongar esta recuperación.

PALABRAS CLAVE Juventud, mercado de trabajo, empleo de los jóvenes, condiciones de trabajo, salarios, género, desempleo, estadísticas del empleo, América Latina 


\section{I}

\section{Introducción}

Los últimos años han sido testigos de una creciente preocupación por el deterioro en las condiciones de inserción de los jóvenes en el mundo del trabajo. Si bien la juventud es un período que se caracteriza por la baja oferta laboral y la alta volatilidad, los sucesivos aumentos en la tasa de desempleo de este grupo etario y la elevada proporción de jóvenes que no estudian y tampoco trabajan han despertado el interés por encontrar las causas detrás de este fenómeno.

De acuerdo con la visión del ciclo de vida laboral, la juventud es un período en que la oferta de trabajo es baja y el empleo es inestable (Breen, 1992). Otro resultado habitual que contribuye a describir la situación de los jóvenes en el mercado laboral es su alta tasa de desempleo. Diversas razones suelen exponerse como determinantes de este resultado. Los cambios demográficos podrían incrementar la oferta relativa de trabajadores jóvenes. Blanchflower y Freeman (2000) evalúan esta hipótesis con respecto a países desarrollados, pero no encuentran evidencia de efectos demográficos en el aumento de la tasa de desempleo juvenil durante los años noventa. En relación con el caso de América Latina, la reducción del crecimiento demográfico en las últimas dos décadas fue una tendencia favorable a la inserción laboral juvenil (Weller, 2006; Fawcett, 2001). Una caída en el nivel educativo de los jóvenes también podría explicar su mayor desempleo. Bell y Blanchflower (2010) encuentran que el nivel educativo juvenil en países industrializados se ha incrementado, mientras que Bassi y Galiani (2009) y Weller (2006) reportan un resultado similar para la región latinoamericana. Otras explicaciones se focalizan en los cambios en la estructura sectorial del empleo en detrimento de aquellos sectores que emplean principalmente a individuos jóvenes, y en las instituciones laborales. La legislación sobre salario mínimo, por ejemplo, puede desalentar la contratación de trabajadores en este rango etario (Neumark y Nizalova, 2007). Asimismo, la incorporación de nuevas tecnologías tiende a acrecentar la demanda de mano de obra calificada pudiendo afectar a la contratación de trabajadores jóvenes (Dolado, Felgueroso y Jimeno, 2000). Otro importante factor que deteriora la inserción

$\square$ Se agradecen los comentarios y las valiosas sugerencias recibidas de parte de un evaluador anónimo en una primera entrega de este trabajo. laboral juvenil en la región es la elevada desigualdad que influye en las oportunidades de acumulación de capital humano y social de los jóvenes (Weller, 2006).

La inestabilidad típica de la inserción laboral de este grupo etario puede ser explicada por distintas teorías. En las de "búsqueda constante de empleo" (job shopping) se sostiene que las transiciones en el mercado de trabajo serían voluntarias. Los jóvenes buscan mejores opciones rotando entre diferentes ocupaciones con un costo de oportunidad bajo (Neumark, 2002). La inestabilidad también podría obedecer a entradas y salidas frecuentes de la fuerza de trabajo para emprender actividades como la acumulación de capital humano. En el otro extremo, la alta rotación laboral juvenil podría ser involuntaria y estar ligada a características individuales y de los puestos de trabajo. Por ejemplo, la menor experiencia laboral de los jóvenes respecto de los adultos redunda en que su situación sea más vulnerable, ya que los costos directos e indirectos de despido serán más bajos en ese caso. Por otra parte, los jóvenes se emplean generalmente en actividades de baja productividad, donde la acumulación de conocimientos específicos es baja (Maurizio, 2011).

En estudios previos se ha mostrado que entre la década de 1990 e inicios de la de 2000 las condiciones económicas y laborales no fueron favorables a la inserción laboral juvenil en la región latinoamericana (Weller, 2006). En el presente trabajo el período de análisis se extiende hasta finales de la década de 2000. La disponibilidad de datos para un período más extenso permitirá analizar la evolución reciente de los indicadores laborales de la población joven. Con esto será posible precisar si en los últimos años se produjo una mejora en sus perspectivas de trabajo o si, por el contrario, el panorama desalentador se mantuvo a pesar de la expansión de las economías latinoamericana durante este período.

Con tal objetivo, en este estudio se realiza un análisis detallado de diversos indicadores laborales para 10 países de la región. Los países seleccionados de acuerdo con la disponibilidad de información son: Argentina, Brasil, Chile, Costa Rica, El Salvador, Honduras, México, Panamá, Uruguay y Venezuela (República Bolivariana de $)^{1}$. Los datos provienen

\footnotetext{
1 Estos países representaron el 74\% de la población total de América Latina en 2012 (Banco Mundial, 2012).
} 
de la Base de Datos Socioeconómicos para América Latina y el Caribe (SEDLAC por sus siglas en inglés), una base de datos de encuestas de hogares de países de América Latina y el Caribe procesada por el Centro de Estudios Distributivos, Laborales y Sociales (CEDLAS) de la Universidad Nacional de La Plata y el Banco Mundial. En el cuadro 1 se aprecia la disponibilidad de información para cada uno de los países y períodos bajo estudio, mientras que en el cuadro A.1 del anexo se precisan las encuestas de hogares utilizadas. La información se divide en seis períodos, separando entre los primeros y los últimos años de cada década. La única excepción es el último período donde también se incluyó información correspondiente a las últimas encuestas disponibles.

A lo largo de este estudio se define como jóvenes al grupo de individuos cuya edad fluctúa entre los 15 y 24 años, mientras que aquellos con edades comprendidas entre los 25 y 65 años serán considerados adultos. Las estadísticas laborales se presentan para el promedio de los 10 países considerados. Este promedio se construye al ponderar el resultado de cada país por su participación en la población total en cada uno de los períodos. A fin de evitar la sobreestimación del peso de aquellos países con mayor disponibilidad de información, se seleccionó un solo año para cada período intentando que la elección sea homogénea entre países (véase el cuadro A.2 del anexo). Lógicamente, en los resultados computados como promedios ponderados se estará dando mayor participación a los países con una población de mayor tamaño. Para los efectos de detectar si los resultados obtenidos están determinados por el comportamiento de estos países, también se reporta el promedio simple y se indica en qué casos los resultados difieren respecto del promedio ponderado.

El artículo se estructura de la siguiente manera. En la sección II se realiza un breve repaso acerca de la evolución de la oferta laboral de los jóvenes en América Latina y sus características. En la sección III se analizan diversos indicadores del mercado de trabajo para jóvenes y adultos a lo largo de las últimas tres décadas, distinguiendo entre hombres y mujeres y entre niveles educativos. En la sección IV se presenta un análisis de pseudo-panel construyendo cohortes de nacimiento con el objetivo de conocer el comportamiento de estos indicadores una vez que los individuos ingresan en la adultez. La sección V concluye con los comentarios finales y las propuestas de política.

CUADRO 1

\section{Encuestas disponibles}

\begin{tabular}{|c|c|c|c|c|c|c|}
\hline País & $\begin{array}{c}\text { Inicios década } \\
1980\end{array}$ & $\begin{array}{c}\text { Finales década } \\
1980\end{array}$ & $\begin{array}{c}\text { Inicios década } \\
1990\end{array}$ & $\begin{array}{c}\text { Finales década } \\
1990\end{array}$ & $\begin{array}{c}\text { Inicios década } \\
2000\end{array}$ & $\begin{array}{l}\text { Finales década } \\
2000\end{array}$ \\
\hline Argentina & 1980 & 1985-1989 & 1990-1994 & 1995-1999 & $2000-2004$ & $2005-2012$ \\
\hline Brasil & $1981-1984$ & $1985-1989$ & 1990, 1992-1993 & 1995-1999 & 2001-2004 & 2005-2009, 2011 \\
\hline Chile & $\ldots$ & 1987 & 1990, 1992, 1994 & 1996, 1998 & 2000,2003 & 2006, 2009, 2011 \\
\hline Costa Rica & $\ldots$ & 1989 & 1990-1994 & 1995-1999 & $2000-2004$ & $2005-2010$ \\
\hline El Salvador & $\ldots$ & $\ldots$ & 1991, 1993 & $\begin{array}{l}1995,1996, \\
1998,1999\end{array}$ & $2000-2004$ & $2005-2010$ \\
\hline Honduras & $\ldots$ & $\ldots$ & 1990-1994 & 1995-1999 & 2001-2004 & $2005-2011$ \\
\hline México & $\ldots$ & 1989 & 1992 & 1996, 1998 & $\begin{array}{l}2000,2002, \\
2004\end{array}$ & $\begin{array}{l}2005-2006 \\
2008,2010\end{array}$ \\
\hline Panamá & $\ldots$ & 1989 & 1991 & $1995,1997,1998$ & 2001-2004 & $\begin{array}{l}\text { 2005-2006, } \\
2009-2012\end{array}$ \\
\hline Uruguay & $\ldots$ & 1989 & 1992 & 1995-1999 & $2000-2004$ & $2005-2011$ \\
\hline $\begin{array}{l}\text { Venezuela (República } \\
\text { Bolivariana de) }\end{array}$ & $\ldots$ & 1989 & 1992 & 1995, 1998, 1999 & 2000-2004 & $2005-2011$ \\
\hline
\end{tabular}

Fuente: elaboración propia sobre la Base de Datos Socioeconómicos para América Latina y el Caribe (SEDLAC) del Centro de Estudios Distributivos, Laborales y Sociales (CEDLAS) y el Banco Mundial. 


\section{II}

\section{Oferta laboral de los jóvenes}

La población joven de América Latina ha mostrado una desaceleración en su ritmo de crecimiento desde inicios de la década de 1980. Mientras que en el período comprendido entre 1980 y 1985 la población juvenil creció un $11 \%$, el aumento fue de solo un $1 \%$ entre 2005 y 2010. Si bien la población en edad de trabajar (entre 15 y 65 años) también se ha acrecentado a una tasa cada vez menor, la desaceleración no fue tan acentuada en este último caso (véase el gráfico 1).

Estos resultados marcan el creciente envejecimiento de la población en edad de trabajar en la región latinoamericana: la participación de los jóvenes se ha reducido del $36 \%$ a inicios de los años ochenta al 27,5\% hacia finales de la década de 2000.

En términos de nivel educativo, la población joven ha mostrado una importante reducción de quienes solo alcanzan al nivel primario. A inicios de los años ochenta, el $72 \%$ de los jóvenes tenía el nivel primario como máximo nivel educativo alcanzado y esa cifra descendió a un $26 \%$ a finales de los años 2000. En tanto que el porcentaje de jóvenes con nivel secundario o superior se incrementó durante las tres décadas analizadas (véase el gráfico 2).

Esta extensión en el período de inversión en capital humano también puede observarse al analizar la distribución de jóvenes de la región según su situación de estudio y empleo. La proporción de jóvenes que solo estudian se ha incrementado en 10 puntos porcentuales a lo largo del período, mientras que el porcentaje de jóvenes que solo trabajan tuvo una reducción similar y el peso de aquellos que no estudian ni trabajan cayó en 5 puntos porcentuales ${ }^{2}$. Como contrapartida, la proporción de los que estudian y laboran aumentó, al igual que el porcentaje de jóvenes desocupados (véase el gráfico 3).

\footnotetext{
2 La proporción de jóvenes que no estudian ni trabajan se ha reducido, pero el tamaño de este grupo es abultado. Cárdenas, de Hoyos y Székely (2011) analizan sus características y las causas de su persistencia.
} GRÁFICO 1

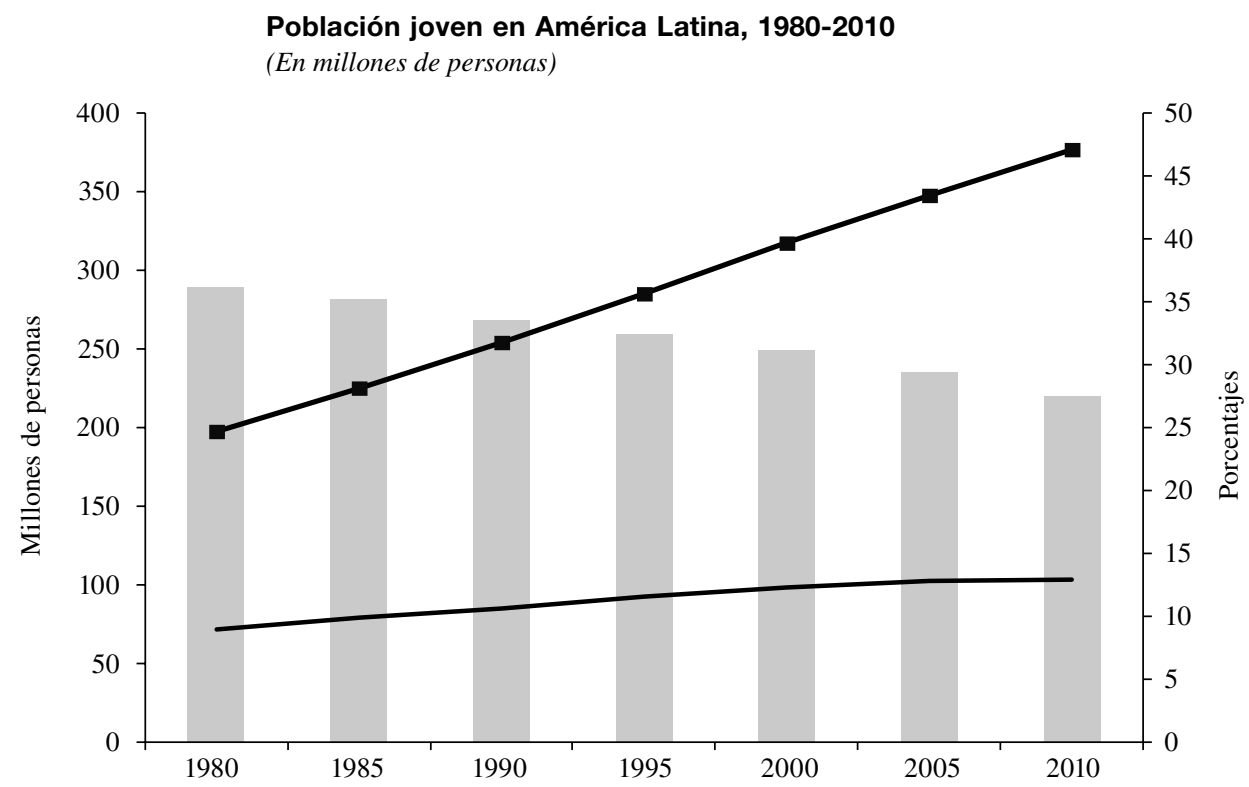

Fuente: elaboración propia sobre la Base de Datos y Publicaciones Estadísticas (CEPALSTAT) de la Comisión Económica para América Latina y el Caribe (CEPAL).

Nota:

Barras grises: población joven como porcentaje de la población en edad de trabajar, intervalos [15-24]/[15-64].

Línea continua: población joven, intervalo [15-24].

Línea con cuadraditos: población en edad de trabajar, intervalo [15-64]. 
GRÁFICO 2

Nivel educativo de la población joven

(Promedio de América Latina)

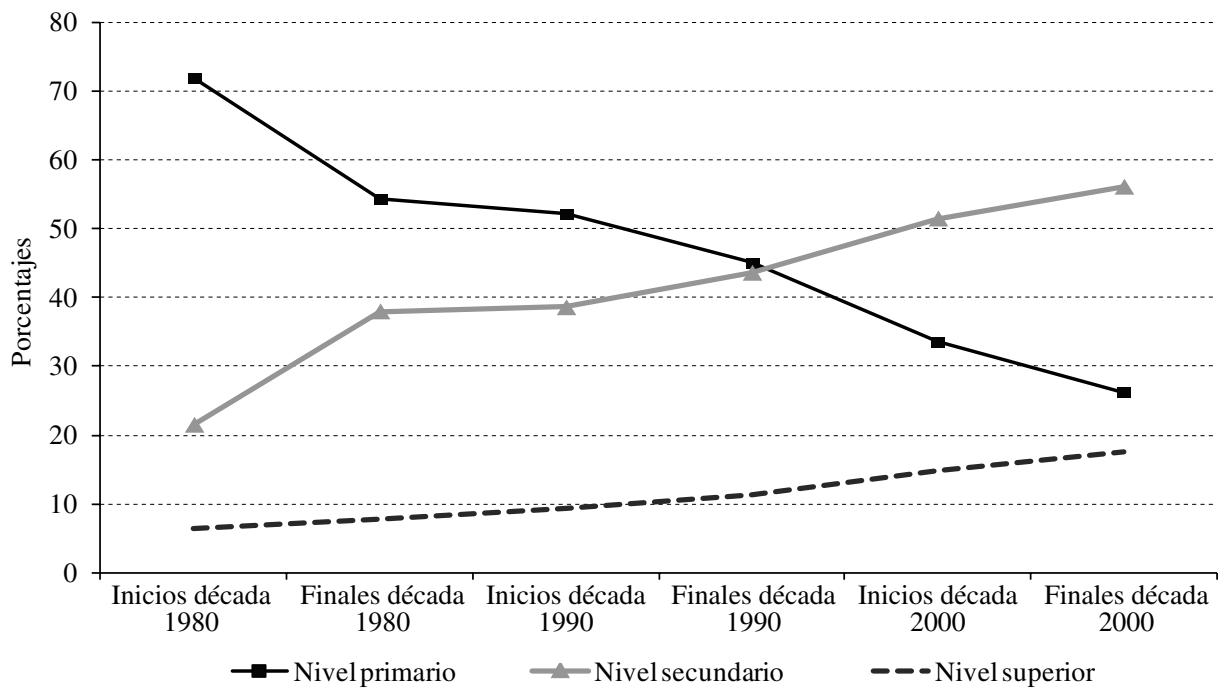

Fuente: elaboración propia sobre la Base de Datos Socioeconómicos para América Latina y el Caribe (sEDLAC) del Centro de Estudios Distributivos, Laborales y Sociales (CEDLAS) y el Banco Mundial.

GRÁFICO 3

Distribución de la población joven según situación de estudios y empleo (Promedio de América Latina)

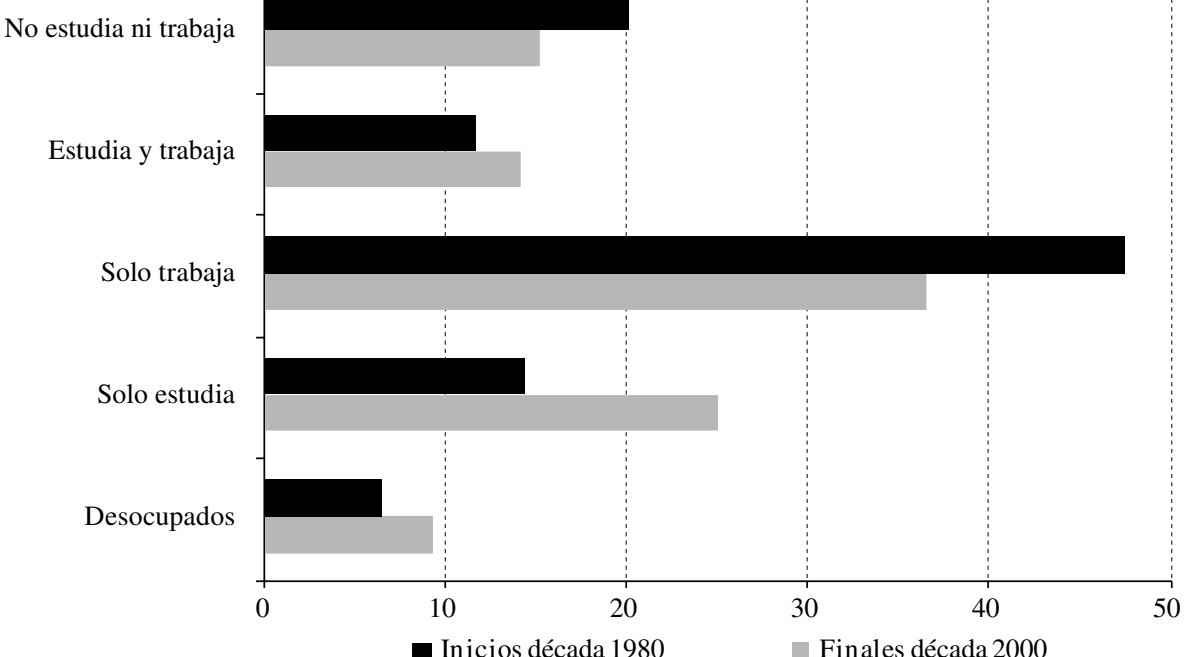

Fuente: elaboración propia sobre la Base de Datos Socioeconómicos para América Latina y el Caribe (SEDLAC) del Centro de Estudios Distributivos, Laborales y Sociales (CEDLAS) y el Banco Mundial.

Esta primera aproximación a la evolución de la oferta laboral de los jóvenes latinoamericanos señala que este grupo etario es cada vez más reducido en relación con la población de la región en edad de trabajar. Su nivel educativo ha mejorado notoriamente en el tiempo, pero a pesar de ello su tasa de desempleo ha tenido una evolución creciente.

La elevada tasa de desempleo juvenil no es una característica exclusiva de la región de América Latina. En otras regiones del mundo se ha registrado 
un empeoramiento en la relación entre desempleo de los jóvenes y desempleo nacional desde inicios de los años noventa (véase el gráfico 4). La región latinoamericana se encontraba en ese momento entre las regiones con valores más bajos respecto de este indicador (el desempleo juvenil superaba al nacional en un $83 \%$ ), pero fue una de las que experimentó un mayor deterioro en esta relación y la que mostró el aumento más notable en la tasa de desempleo de los jóvenes.

\section{A. $1990-1994$}

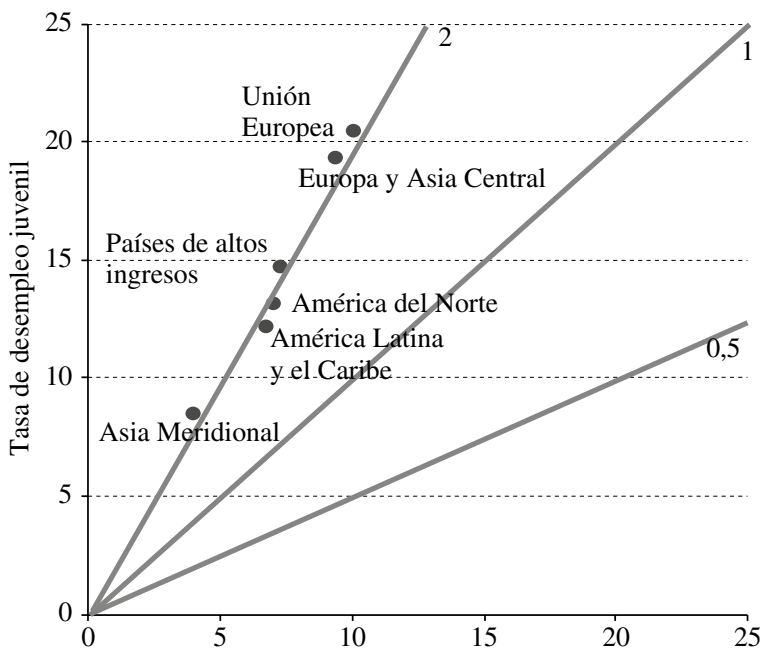

B. $2005-2009$

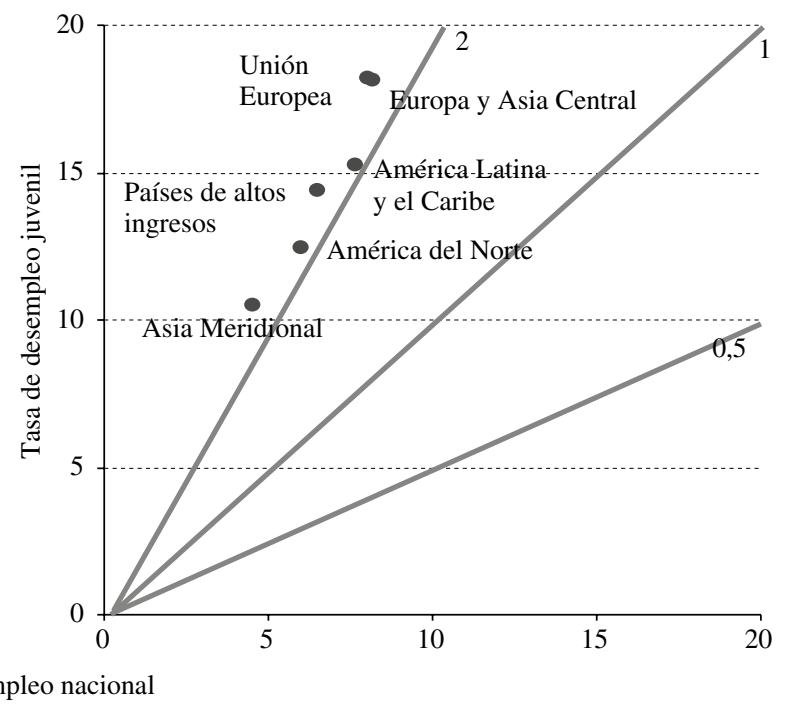

Fuente: elaboración propia sobre la base de Banco Mundial, World Development Indicators.

\section{III}

\section{Situación laboral de los jóvenes en América Latina}

\section{Participación e inserción laboral de los jóvenes}

Los jóvenes latinoamericanos han visto reducida su participación laboral desde inicios de los años ochenta. En los adultos el comportamiento fue el opuesto, lo que determinó que la brecha de participación se ampliara durante el período (véase el cuadro A.3 del anexo). En el primer panel del gráfico 5 se evidencia la evolución de este indicador, calculado como un promedio ponderado. En el primer período solo se cuenta con información para la Argentina y el Brasil, mientras que respecto de los finales de la década de 1980 la muestra se amplía e incluye a Chile, Costa Rica, México, Panamá, el Uruguay y Venezuela (República Bolivariana de). Con el objeto de evaluar qué proporción del cambio entre inicios y finales de esa década puede explicarse por la ampliación de la muestra, las líneas punteadas denotan el valor del indicador a fines de los años ochenta si solo la Argentina y el Brasil hubiesen sido observados. La ampliación de la muestra se traduce en una caída de la tasa de actividad de los jóvenes. Esto puede explicarse por la incorporación de Chile y Venezuela (República Bolivariana de), países 
con tasas de participación juvenil muy por debajo de las registradas en la Argentina y el Brasil con respecto a este período $^{3}$. El valor computado como el promedio simple indica un nivel y una evolución similares en el caso de los adultos y un patrón decreciente con un nivel menor en el de los jóvenes (véase el panel correspondiente a promedio simple del gráfico 5).

Una posible explicación del comportamiento decreciente en la tasa de participación juvenil puede encontrarse en la prolongación del período de inversión en capital humano. Como se señaló en la sección II, la proporción de jóvenes que solo estudian aumentó un $67 \%$ durante el período, representando un cuarto de la población juvenil de América Latina hacia fines de la década de 2000. Como resultado, el porcentaje de jóvenes con nivel primario se redujo y la proporción con nivel secundario y superior mostró un incremento. En lo que se refiere a los adultos, el aumento en su tasa de participación puede explicarse por la incorporación de las mujeres al mercado laboral. En el cuadro A.4 del anexo se constata que la tasa de participación de los hombres adultos se mantuvo estable en alrededor del $91 \%$, mientras que la de las mujeres creció del $40 \%$ a

3 Las estadísticas por país se encuentran disponibles y pueden ser solicitadas a la autora. inicios de los años ochenta al $61 \%$ a finales de los años 2000. Entre los jóvenes, la desagregación por género deja ver que la caída en la tasa de participación de este grupo ocurrió principalmente entre los hombres, mientras que entre las mujeres creció levemente.

La restricción de la muestra al grupo de individuos que no asiste a la educación formal permite controlar por el fenómeno de extensión en el período de inversión en capital humano anteriormente descrito (véase el cuadro A.5 del anexo). En este caso la participación de los jóvenes no registró grandes variaciones promediando el $73 \%$ y fue superior a la tasa reportada para la totalidad de los jóvenes, reflejando el fenómeno de competencia entre el estudio y el trabajo (Marchionni, Bet y Pacheco, 2007), e incluso fue mayor a la tasa de participación de los adultos durante los años ochenta y noventa. De aquí se desprende que entre los jóvenes que no estudian, alrededor del $30 \%$ tampoco trabajan ni buscan empleo activamente. La diferencia de nivel entre hombres y mujeres evidencia que la tasa de participación se mantuvo en alrededor del $90 \%$ para los primeros, y creció del $50 \%$ al $60 \%$ para las mujeres. Esta discrepancia, sin duda, tiene que ver con la realización de actividades no remuneradas típicamente domésticas por parte de las mujeres, junto con el desempeño de responsabilidades familiares.

Un comportamiento similar al de la tasa de participación se observa en la evolución de la tasa de
A. Promedio ponderado

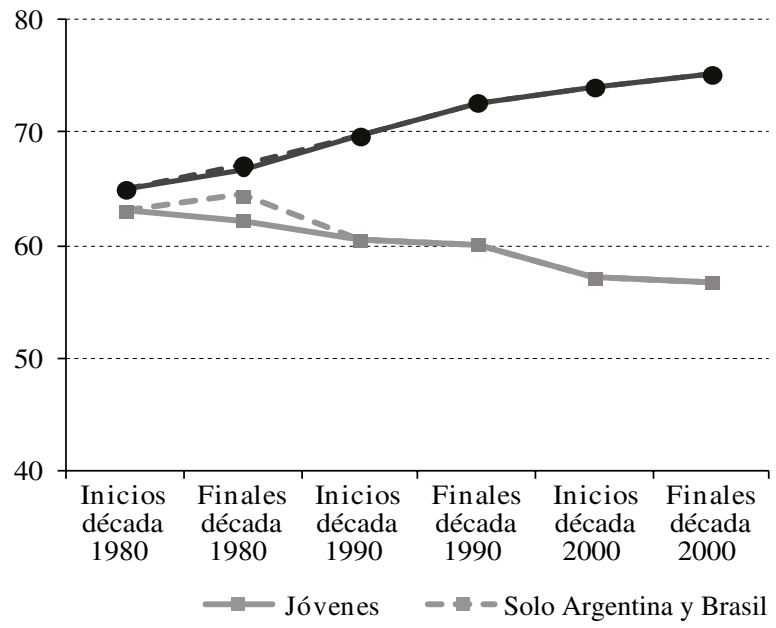

B. Promedio simple

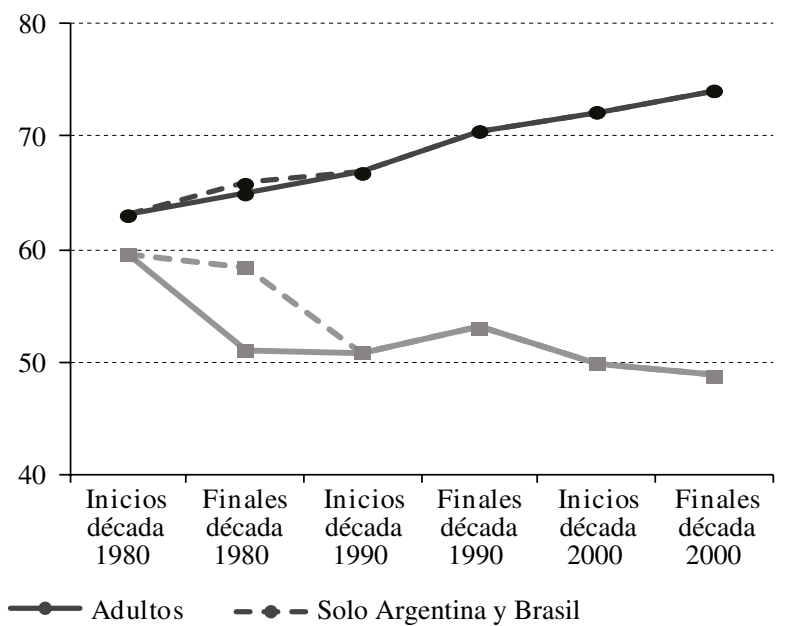

Fuente: elaboración propia sobre la Base de Datos Socioeconómicos para América Latina y el Caribe (sedLaC) del Centro de Estudios Distributivos, Laborales y Sociales (CEDLAS) y el Banco Mundial. 
empleo de los jóvenes (véase el gráfico 6). El nivel de empleo de este grupo denotó una clara caída a lo largo del período y lo opuesto ocurrió entre los adultos, aunque cabe destacar la leve recuperación en la tasa de empleo juvenil entre inicios y finales de la década de 2000. Nuevamente, la incorporación de países a la muestra hacia finales de los años ochenta se traduce en una menor tasa de empleo con respecto a la que surge de considerar solo a la Argentina y el Brasil. En consecuencia, el cálculo como un promedio simple muestra un nivel de empleo inferior.

La caída en la participación laboral de los jóvenes permitió plantear la hipótesis de una mayor inversión en capital humano, pero ¿qué sucedió con aquellos que permanecieron en el mercado de trabajo y no estudian? Cuando la muestra se restringe a los individuos que no asisten a un establecimiento educativo, el nivel de empleo juvenil es superior respecto del registrado para la totalidad de este grupo y también se observa un descenso a lo largo del tiempo. Aquí cabe hacer una observación importante. $\mathrm{Al}$ reducir la muestra, excluyendo a los individuos que estudian, la tasa de participación de los jóvenes pasa de ser decreciente a estable, mientras que la tasa de empleo mantiene su patrón a la baja, aunque menos pronunciado, con una recuperación entre inicios y finales de la década de 2000. La distinción por niveles educativos indica que la caída del empleo fue más aguda entre los jóvenes de los niveles primario y secundario respecto de los de nivel superior (véase el cuadro A.7 del anexo). En el

GRÁFICO 6

Tasa de empleo

(Promedio de América Latina)

A. Promedio ponderado

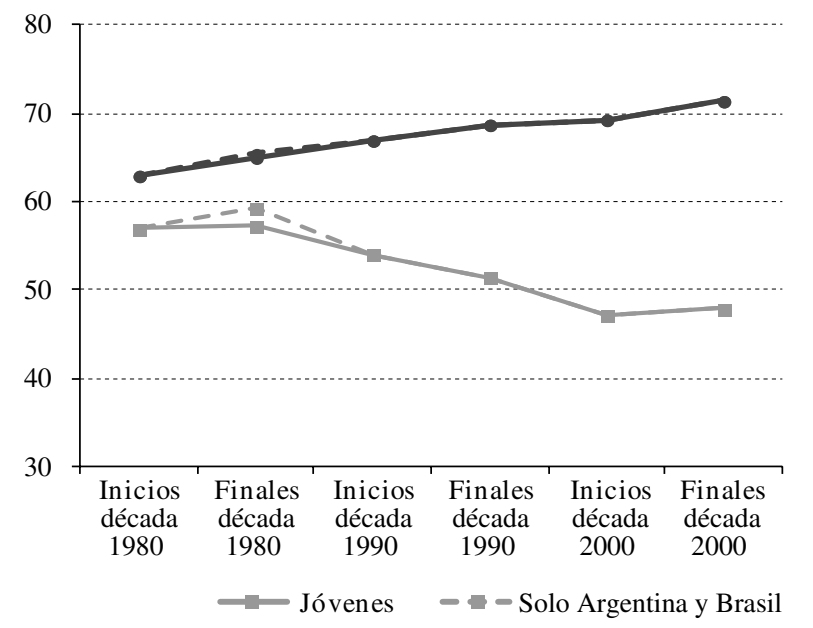

último período estas tendencias generales se modifican: el deterioro del empleo de los de menor nivel educativo se detiene, en los jóvenes de nivel secundario se produce una recuperación y en los de nivel superior, una pequeña merma. La desagregación por género permite ver que la caída del empleo solo existió entre los hombres, y que entre las mujeres se verificó un aumento, especialmente en el último período. Esta evidencia es un claro indicio del incremento en el número de jóvenes desempleados entre aquellos que no se encuentran acumulando capital humano, con una posible recuperación hacia finales del período bajo estudio.

La evolución de la tasa de desempleo juvenil pone de manifiesto que este es un problema central en los mercados laborales de la región. Entre finales de los años ochenta e inicios de los años 2000 la tasa de desempleo de los jóvenes se duplicó. A partir de entonces comenzó a descender, al igual que la tasa de desempleo adulta (véase el gráfico 7). Al calcular el promedio simple se observa que la ampliación de la muestra a finales de los años ochenta produjo un cambio brusco. Esto obedece a la elevada tasa de desempleo juvenil en países "pequeños" como Chile, Panamá y el Uruguay. Hacia inicios de los años noventa mejoró el valor de este indicador en estos países, dando lugar a una trayectoria similar a la obtenida con el promedio ponderado por tamaño de población, aunque con un nivel superior.

Detrás de estos valores promedio se encuentran experiencias muy heterogéneas entre países. Sin embargo,

Fuente: elaboración propia sobre la Base de Datos Socioeconómicos para América Latina y el Caribe (SEDLAC) del Centro de Estudios Distributivos, Laborales y Sociales (CEDLAS) y el Banco Mundial.

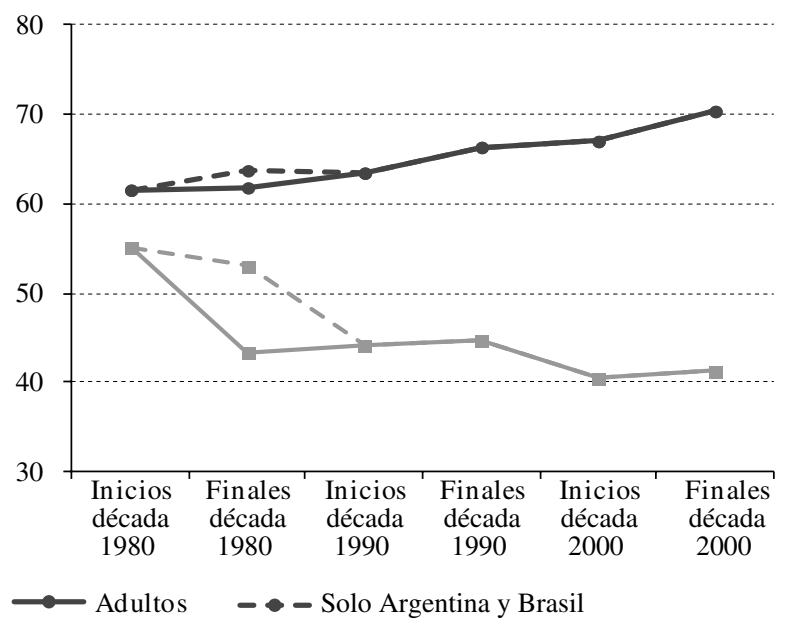


A. Promedio ponderado

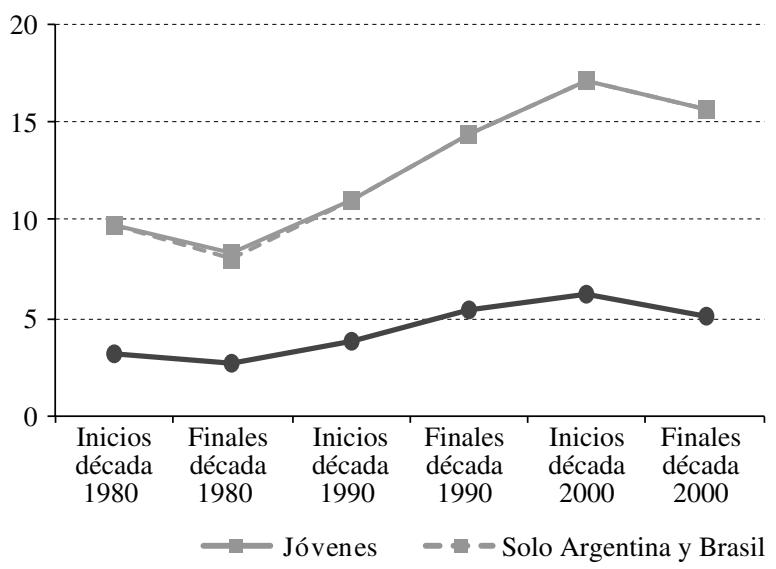

B. Promedio simple

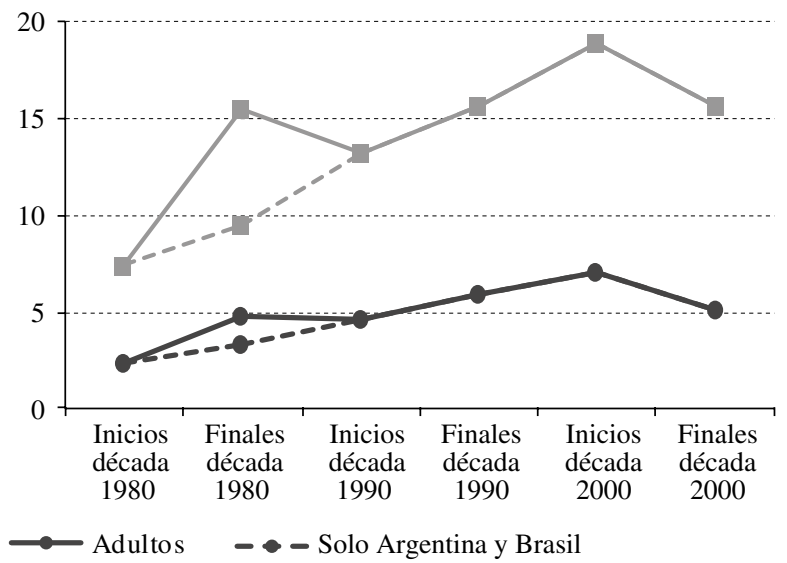

Fuente: elaboración propia sobre la Base de Datos Socioeconómicos para América Latina y el Caribe (SEDLAC) del Centro de Estudios Distributivos, Laborales y Sociales (cedlas) y el Banco Mundial.

la comparación entre las tasas de desempleo nacional y juvenil en el último período indica que el desempleo de los jóvenes duplica con creces el total nacional en 7 de los 10 países. Honduras, El Salvador y Venezuela (República Bolivariana de) son la excepción, con tasas de desempleo juvenil que hacia finales de la década de 2000 fueron $78 \%, 88 \%$ y $92 \%$ superiores a la de los adultos, respectivamente. En todos los países, los jóvenes representan entre el 37\% (República Bolivariana de Venezuela) y el 54\% (Honduras) del total de desempleados (véase el gráfico 8).

Cuando el análisis se restringe a la muestra de individuos que no asisten a un establecimiento educativo es posible aislar los casos en que el desempleo ocurre por la realización de una actividad que compite con el tiempo dedicado al trabajo (el estudio). En este caso se encuentra una evolución similar en la tasa de desempleo juvenil con un nivel inferior respecto de la muestra completa. La distinción por género indica que la incidencia del desempleo es menor entre los hombres. El análisis por niveles educativos destaca algunos patrones: i) la tasa de desempleo tuvo una evolución creciente para todos los niveles educativos con una recuperación a finales de los años 2000; ii) el desempleo juvenil muestra un patrón de $\mathrm{U}$ invertida en función del nivel de educación para todos los períodos (véase el gráfico 9), y iii) el desempleo de los jóvenes de nivel superior es levemente mayor que el de los de nivel primario, con excepción de la década de 1980.
En relación con esta última observación pueden haber dos posibles explicaciones. La primera de ellas tiene que ver con un componente involuntario de la rotación laboral juvenil. Los empleos que requieren un alto nivel de calificación también pueden demandar experiencia en el desempeño de esas tareas y será más difícil para los jóvenes cumplir con ese requisito. La inserción de jóvenes de alto nivel educativo también puede ser difícil debido a la baja productividad característica de las actividades con mayor participación juvenil (Maurizio, 2011). La segunda explicación se vincula a un componente voluntario de la rotación laboral. La búsqueda de la mejor coincidencia (matching) puede ser más prolongada para los jóvenes más educados, quienes intentarán encontrar un puesto de trabajo acorde con su calificación.

La relación de $\mathrm{U}$ invertida entre la tasa de desempleo juvenil y el nivel educativo podría deberse al aumento en la oferta de jóvenes con nivel secundario (véase el gráfico 2). Pero esta relación se hace presente en todos los períodos y no solo en los años 2000 cuando la proporción de jóvenes con nivel secundario superó a la de nivel primario. La explicación parecería radicar en la demanda. Las actividades que emplean principalmente a trabajadores jóvenes suelen ser de baja productividad y demandarán individuos de bajo nivel educativo. La demanda de trabajadores jóvenes de mayor calificación será menor, pero también lo será su oferta. Los trabajadores de nivel secundario quedarían entonces segmentados, con una demanda baja y una oferta en crecimiento. 
GRÁFICO 8

Desempleo nacional comparado con desempleo juvenil

(Finales década 2000)

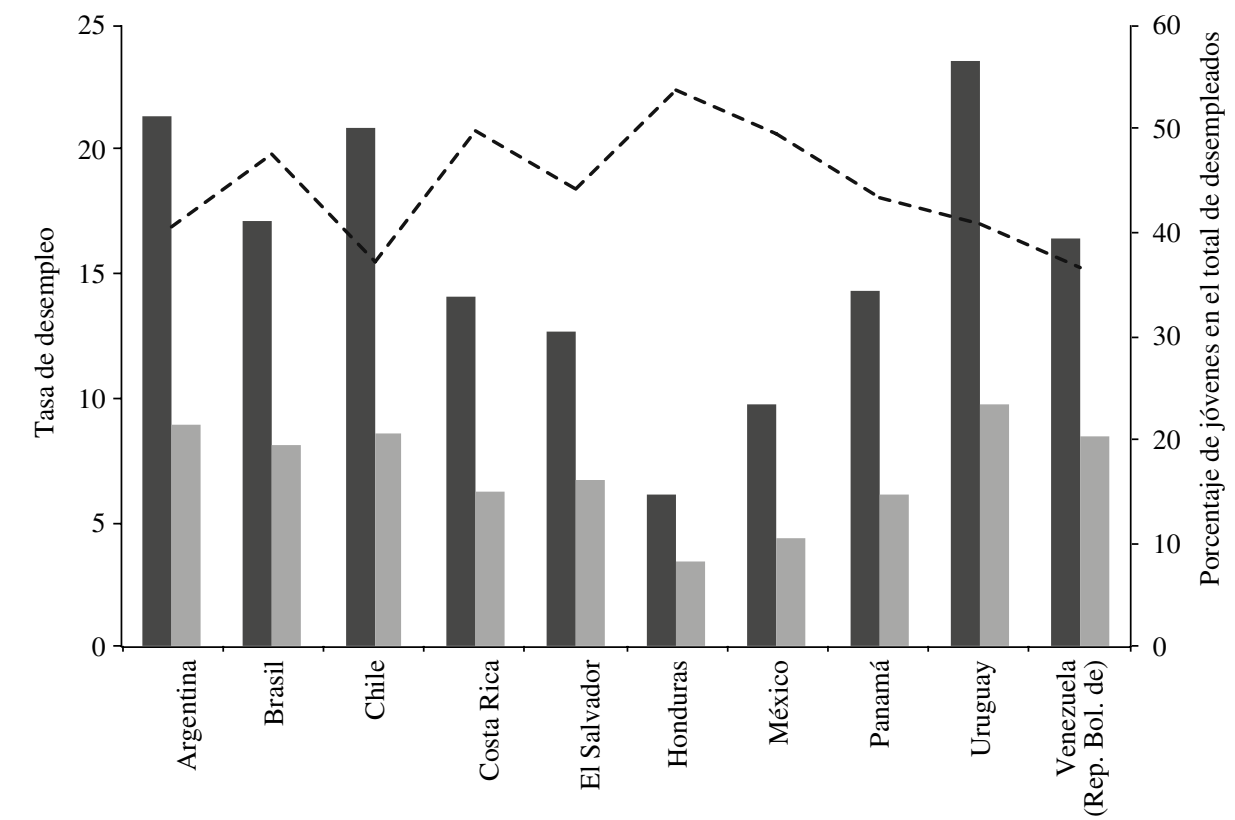

Desempleo juvenil Desempleo nacional - - - Porcentaje de jóvenes en el total de desempleados

Fuente: elaboración propia sobre la Base de Datos Socioeconómicos para América Latina y el Caribe (sEDLAC) del Centro de Estudios Distributivos, Laborales y Sociales (CEDLAs) y el Banco Mundial.

GRÁFICO 9

Tasa de desempleo según niveles educativos

(Individuos que no asisten a la educación formal)

A. Promedio ponderado

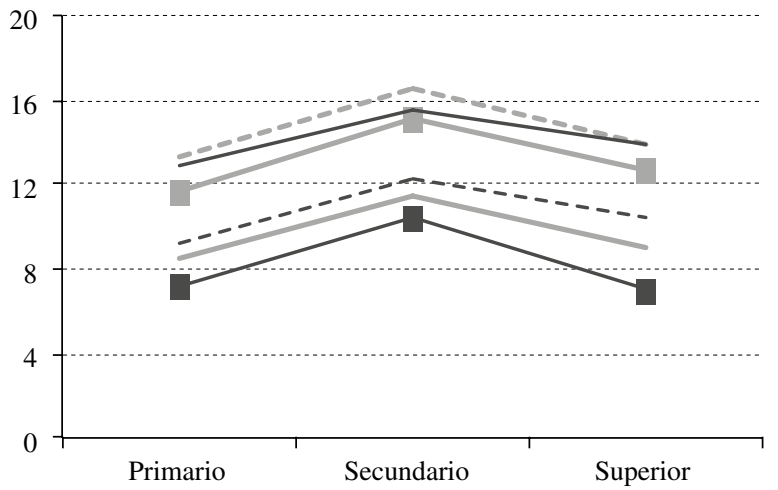

— Inicios década $1980 \quad$ - - - - Inicios década 1990

—_ Finales década $1980 \quad$ - Finales década 1990

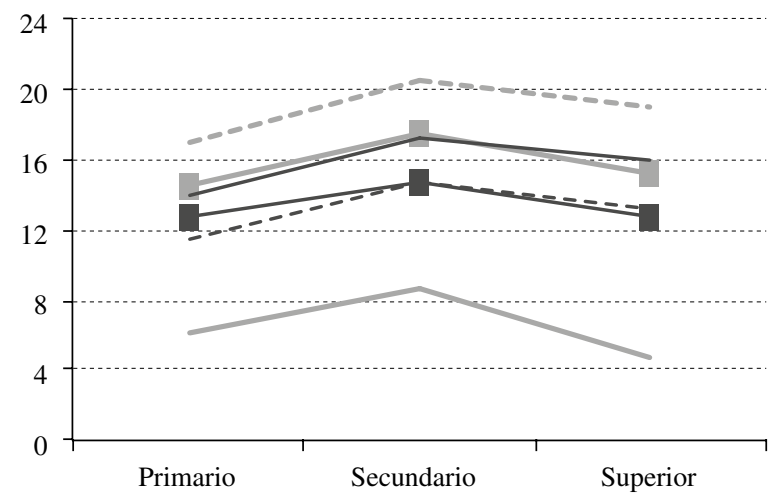

- - - Inicios década 2000

- Finales década 2000

Fuente: elaboración propia sobre la Base de Datos Socioeconómicos para América Latina y el Caribe (sEDLAC) del Centro de Estudios Distributivos, Laborales y Sociales (CEDLAS) y el Banco Mundial. 
La extensión del período de desempleo es superior entre los adultos. En el gráfico 10 se aprecia la distribución de desocupados en tres categorías: hasta 6 meses de desempleo, entre 6 meses y un año, y más de 1 año buscando trabajo. Durante los años ochenta los jóvenes desocupados se concentraron en las primeras dos categorías y a partir de los años noventa la distribución se polarizó entre las categorías de desempleo de menor y mayor duración. La distribución de adultos desocupados tiene un patrón similar con una fracción mayor en la categoría de desempleo de más larga duración en comparación con los jóvenes. Este resultado parece contraintuitivo dada la desventaja de los jóvenes en el momento de buscar un trabajo debido a su menor experiencia laboral acumulada. Sin embargo, es importante tener en cuenta que la duración del desempleo se reporta en el momento de la encuesta y no se conoce la duración total del episodio; con respecto a los trabajadores jóvenes con un breve historial en el mercado de trabajo, la duración del desempleo también estará determinada por esa condición. Otra posible razón detrás de esta brecha puede encontrarse en las distintas preferencias cuando se busca trabajo. Los jóvenes que recién ingresan al mercado laboral no conocen completamente los puestos disponibles y rotan entre distintas ocupaciones con períodos de desempleo entre cada una de ellas que pueden ser breves. El mayor conocimiento y las preferencias más claras de los adultos pueden generar períodos de desempleo más extensos hasta lograr la mejor coincidencia con la opción deseada.

\section{Caracterización del empleo de los jóvenes}

El porcentaje de individuos que desean cambiar de empleo o trabajar más horas es un indicador subjetivo de la situación laboral de los trabajadores. Su valor siempre ha sido superior entre los jóvenes en el promedio de América Latina, aunque el nivel y la evolución de este indicador difiere de acuerdo con el país considerado. En el Brasil, Costa Rica, Honduras y Venezuela (República Bolivariana de) la tendencia ha sido creciente, en Panamá el comportamiento fue el opuesto, en la Argentina y el Uruguay este indicador aumentó hasta finales de la década de 2000 y a partir de entonces comenzó a descender ${ }^{4}$.

La tasa de informalidad - definida como el porcentaje de puestos no registrados en la seguridad social - siempre ha sido superior entre los jóvenes, con una diferencia promedio respecto de los adultos de 20 puntos porcentuales. La evolución fue creciente para ambos grupos de edad con una pequeña recuperación para los jóvenes hacia finales de los años 2000. El análisis de las implicancias de la informalidad sobre el bienestar de los trabajadores jóvenes resulta difícil dada la definición utilizada. Si estos trabajadores continúan estando a cargo de un adulto (del jefe del hogar, por ejemplo) podrán acceder a la cobertura social si ese individuo es

\footnotetext{
4 En Chile, México y El Salvador no se dispone de información acerca de este indicador.
} GRÁFICO 10

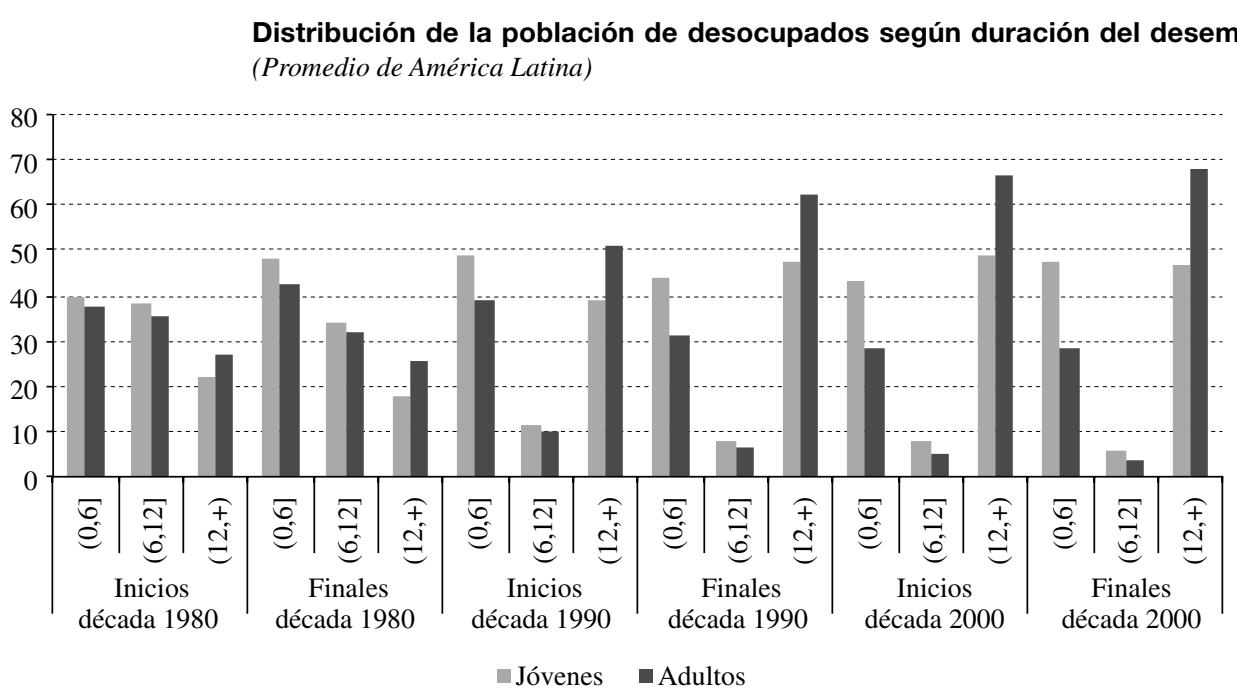

Fuente: elaboración propia sobre la Base de Datos Socioeconómicos para América Latina y el Caribe (SEDLAC) del Centro de Estudios Distributivos, Laborales y Sociales (CEDLAs) y el Banco Mundial.

Nota: $(x, y)$ intervalo sin incluir ambos extremos $x$ e $y$.

$(\mathrm{x}, \mathrm{y}]$ intervalo excluye extremo inferior $\mathrm{x}$ e incluye extremo superior $\mathrm{y}$. 
un trabajador formal ${ }^{5}$. Más allá de esto, la condición de informalidad en la juventud puede afectar a la inserción laboral en el futuro, aumentando las posibilidades de empleo informal en la adultez o reduciendo el salario si la informalidad es interpretada como una señal de baja productividad (Cruces, Ham y Viollaz, 2012).

Existe una clara relación negativa entre la tasa de informalidad y el nivel educativo (véase el cuadro A.6 del anexo). Los trabajadores jóvenes de nivel primario se encuentran particularmente desprotegidos: la diferencia en la tasa de informalidad entre los jóvenes de nivel primario y secundario es de 26 puntos porcentuales, y en relación con los de nivel superior la diferencia asciende a 37 puntos. Asimismo, la caída en la tasa de informalidad juvenil observada hacia finales de la década de 2000 se explica enteramente por el retroceso registrado en el grupo de nivel superior.

El perfil creciente en la tasa de desempleo juvenil junto con una tasa de informalidad en aumento sugieren que los jóvenes ingresarían al sector informal ante la existencia de algún tipo de barrera de entrada al sector formal, que es particularmente infranqueable para los de menores niveles educativos. La evidencia de niveles crecientes de calificación conduce a rechazar las brechas educativas como explicación de una mayor tasa de informalidad entre los jóvenes. Por el contrario, se estaría dando un proceso de segregación ocupacional donde los puestos no cubiertos por la seguridad social recaen sobre todo en trabajadores jóvenes.

El salario horario (en dólares a paridad de poder de compra del año 2005) siempre ha sido superior entre los adultos. Este es, sin duda, un resultado esperado, ya que la mayor edad se traduce inmediatamente en una experiencia laboral más prolongada. El diferencial salarial alcanzó su máximo hacia finales de los años noventa cuando un trabajador adulto ganó, en promedio, 2 dólares más que uno joven. La diferencia de salario entre jóvenes y adultos se amplía con la educación: la brecha promedio fue inferior a 1 dólar en el nivel primario, alcanzó a los 2 dólares en el secundario y trepó a 5 dólares en el nivel superior. Otra observación interesante es la mejora relativa de los jóvenes con menor educación para el promedio de la región.

El diferencial salarial por niveles educativos entre los jóvenes muestra una evolución distinta de acuerdo con el país considerado (véase el cuadro 2).

\footnotetext{
5 En la mayoría de los países de América Latina, un trabajador formal puede extender la cobertura de la seguridad social — por ejemplo, el seguro de salud - a los miembros de su familia, especialmente a su cónyuge e hijos.
}

En la Argentina, Chile y Costa Rica el diferencial osciló alrededor de 1,4. El Uruguay es el único país donde esta razón aumentó con el transcurso del tiempo. El Brasil, El Salvador y Panamá mostraron tendencias decrecientes y, por último, México y Venezuela (República Bolivariana de) experimentaron etapas sucesivas de crecimiento y retraimiento de la tasa salarial entre jóvenes calificados y no calificados.

Las horas semanales de trabajo de jóvenes y adultos cayeron a lo largo del período, con un descenso en el grupo juvenil un poco más pronunciado. A inicios de los años ochenta la brecha de horas de trabajo era de 0,7 horas en favor de los adultos, mientras que a fines de la década de 2000 alcanzó a 2,1 horas. Los individuos de nivel educativo superior, jóvenes y adultos, trabajan menos horas en promedio que los de nivel primario y secundario.

\section{Resultados laborales de los jóvenes desde una perspectiva de género}

Las características de la inserción laboral difieren entre hombres y mujeres, al igual que los resultados que ellos obtienen en el mercado de trabajo. En general, las mujeres enfrentan condiciones laborales desventajosas debido a la dificultad de combinar el trabajo remunerado con el que se realiza en el hogar, y a los fenómenos de discriminación y segregación por género presentes en los mercados laborales latinoamericanos (Maurizio, 2010).

Como se describió anteriormente, la caída de la tasa de participación de los jóvenes a lo largo del tiempo se explica por la menor actividad de los hombres. Por el contrario, las mujeres se han ido incorporando al mercado de trabajo, aunque su participación sigue siendo menor que la de los hombres. En cuanto al empleo, tanto los hombres como las mujeres jóvenes poseen tasas de empleo cada vez menores.

En este punto es importante analizar la distribución de hombres y mujeres jóvenes según su situación de estudio y empleo. El gráfico 11 muestra que la distribución difiere notablemente por género, a pesar de que la evolución de cada categoría ha ido en igual sentido. Entre los hombres jóvenes la categoría que domina es la de "solo trabaja", que pasó de $63 \%$ a inicios de los años ochenta a $46 \%$ a finales de la década de 2000. Entre las mujeres las categorías dominantes son las de "solo trabaja" y "no estudia ni trabaja"; estos porcentajes variaron de sobre el $30 \%$ a alrededor del $25 \%$ a fines del período. Como contrapartida, aumentó la proporción de mujeres jóvenes que solo estudian y el porcentaje de 
Jóvenes: diferencial salarial por nivel educativo.

Inicios década 1980 - finales década 2000

(Cocientes ${ }^{\mathrm{a}}$ )

\begin{tabular}{|c|c|c|c|c|c|c|}
\hline & $\begin{array}{c}\text { Inicios década } \\
1980\end{array}$ & $\begin{array}{c}\text { Finales década } \\
1980\end{array}$ & $\begin{array}{c}\text { Inicios década } \\
1990\end{array}$ & $\begin{array}{c}\text { Finales década } \\
1990\end{array}$ & $\begin{array}{c}\text { Inicios década } \\
2000\end{array}$ & $\begin{array}{c}\text { Finales década } \\
2000\end{array}$ \\
\hline \multicolumn{7}{|l|}{ Argentina } \\
\hline Media & $\ldots$ & 1,417 & 1,305 & 1,401 & 1,385 & 1,399 \\
\hline Error estándar & $\ldots$ & 0,005 & 0,002 & 0,001 & 0,002 & 0,001 \\
\hline \multicolumn{7}{|l|}{ Brasil } \\
\hline Media & 2,444 & 2,476 & 2,236 & 1,905 & 1,696 & 1,490 \\
\hline Error estándar & 0,001 & 0,002 & 0,001 & 0,001 & 0,001 & 0,001 \\
\hline \multicolumn{7}{|l|}{ Chile } \\
\hline Media & $\ldots$ & $\ldots$ & 1,396 & 1,398 & 1,244 & 1,321 \\
\hline Error estándar & $\ldots$ & $\ldots$ & 0,004 & 0,007 & 0,003 & 0,004 \\
\hline \multicolumn{7}{|l|}{ Costa Rica } \\
\hline Media & $\ldots$ & 1,486 & 1,491 & 1,477 & 1,577 & 1,435 \\
\hline Error estándar & $\ldots$ & 0,008 & 0,003 & 0,004 & 0,004 & 0,002 \\
\hline \multicolumn{7}{|l|}{ El Salvador } \\
\hline Media & $\ldots$ & $\ldots$ & 1,996 & 1,599 & 1,374 & 1,401 \\
\hline Error estándar & $\ldots$ & $\ldots$ & 0,022 & 0,003 & 0,002 & 0,002 \\
\hline \multicolumn{7}{|l|}{ Honduras } \\
\hline Media & $\ldots$ & $\ldots$ & 2,298 & 1,878 & 1,924 & 1,782 \\
\hline Error estándar & $\ldots$ & $\ldots$ & 0,009 & 0,004 & 0,005 & 0,005 \\
\hline \multicolumn{7}{|l|}{ México } \\
\hline Media & $\ldots$ & $\ldots$ & 1,887 & 1,724 & 1,935 & 1,518 \\
\hline Error estándar & $\ldots$ & $\ldots$ & 0,003 & 0,001 & 0,002 & 0,001 \\
\hline \multicolumn{7}{|l|}{ Panamá } \\
\hline Media & $\ldots$ & $\ldots$ & 1,693 & 1,519 & 1,566 & 1,420 \\
\hline Error estándar & $\ldots$ & $\ldots$ & 0,012 & 0,008 & 0,004 & 0,003 \\
\hline \multicolumn{7}{|l|}{ Uruguay } \\
\hline Media & $\ldots$ & 1,227 & 1,256 & 1,292 & 1,346 & 1,410 \\
\hline Error estándar & $\ldots$ & 0,006 & 0,007 & 0,004 & 0,004 & 0,004 \\
\hline \multicolumn{7}{|c|}{ Venezuela (República Bolivariana de) } \\
\hline Media & $\ldots$ & 1,447 & 1,539 & 1,783 & 1,567 & 1,218 \\
\hline Error estándar & $\ldots$ & 0,002 & 0,003 & 0,004 & 0,007 & 0,001 \\
\hline
\end{tabular}

Fuente: elaboración propia sobre la Base de Datos Socioeconómicos para América Latina y el Caribe (SEDLAC) del Centro de Estudios Distributivos, Laborales y Sociales (CEDLAS) y el Banco Mundial.

a Cocientes entre el salario horario de jóvenes calificados (nivel educativo secundario completo o superior) y el de no calificados (nivel educativo secundario incompleto o inferior).

desempleadas. Más allá de este cambio de tendencias, la proporción de mujeres jóvenes que solo realizan actividades domésticas sigue sobrepasando con creces el valor registrado para los hombres.

El creciente desempleo juvenil ha afectado de modo similar a hombres y mujeres, aunque la tasa de desempleo de estas últimas siempre ha superado la de los hombres. En cuanto a las características de los empleos, hombres y mujeres jóvenes han tenido tasas de informalidad de alrededor del $50 \%$, pero los hombres fueron más afectados por el incremento de este indicador entre finales de los años ochenta e inicios de los años 2000. El salario horario mostró una tendencia al alza en ambos casos y esta recuperación fue más pronunciada entre las mujeres jóvenes, permitiendo que la brecha salarial se cerrara hacia inicios de la década de 2000. 
A. Hombres

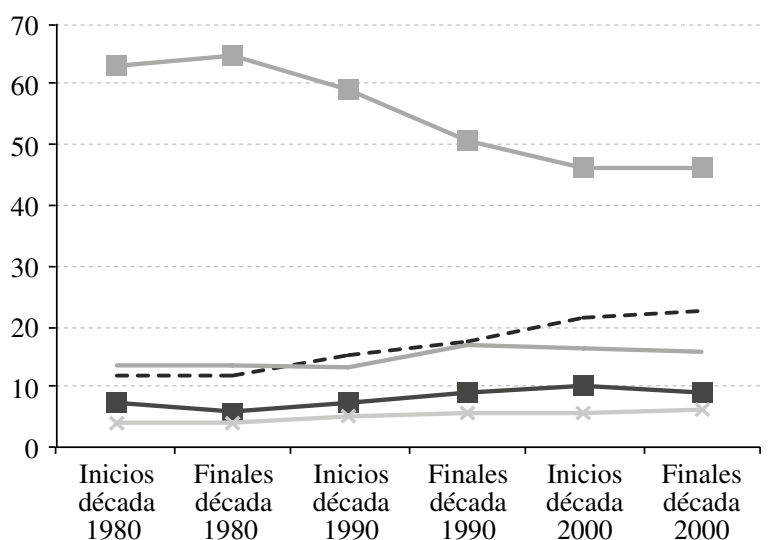

$\longrightarrow$ Desocupados -----Solo estudia $\quad-$ Solo trabaja
B. Mujeres

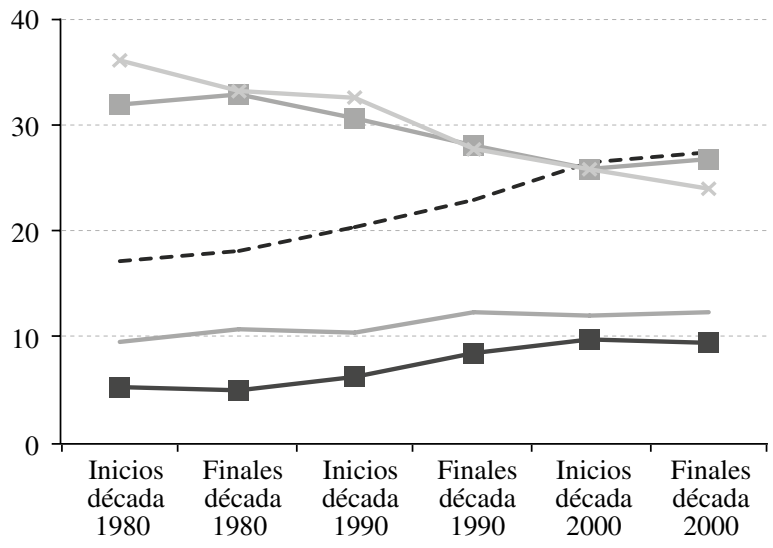

Fuente: elaboración propia sobre la Base de Datos Socioeconómicos para América Latina y el Caribe (sEdLac) del Centro de Estudios Distributivos, Laborales y Sociales (CEDLAs) y el Banco Mundial.

\section{IV}

\section{Análisis del ciclo de vida laboral}

Esta sección tiene por objetivo analizar la dinámica de los mercados de trabajo de América Latina, evaluando el comportamiento de indicadores laborales a lo largo del ciclo de vida. Con ello se procura encontrar patrones de comportamiento que no se pueden detectar en un análisis de corte transversal. Por ejemplo, si las dificultades que los jóvenes encuentran al incorporarse al mercado de trabajo persisten una vez que llegan a la adultez, y si esta relación se ha modificado con el transcurso del tiempo. La inestabilidad laboral que enfrentan los jóvenes en sus primeros años en el mercado de trabajo podría repercutir en sus resultados laborales futuros. Por ejemplo, es posible que los jóvenes que estuvieron expuestos a elevadas tasas de desempleo sufran una "penalidad" al tener mayores posibilidades de estar desempleados durante la adultez.

La información disponible no posee una estructura de panel. La propuesta metodológica consiste en definir cohortes de nacimiento y seguir su comportamiento a lo largo del tiempo (véase el cuadro 3). De acuerdo con la disponibilidad de información para los 10 países considerados, se construyeron 6 cohortes. La primera de ellas está compuesta por individuos nacidos en la primera mitad de la década de 1960. Estos individuos serán observados desde sus 16 años y hasta los 49 años. La segunda cohorte corresponde a los individuos nacidos en la segunda mitad de la misma década que podrán ser observados desde sus 15 años y hasta los 44 años. Las tercera, cuarta y quinta cohorte se construyen de manera similar. Sus integrantes serán observados desde los 15 años y hasta cierta edad en la adultez. El período de observación será más corto en la medida en que se consideran cohortes más actuales. Los individuos nacidos en la segunda mitad de los años ochenta conforman la sexta y última cohorte, que solo es observada durante su juventud. Los promedios para toda la región se calcularon ponderando por la participación de cada país en el subgrupo de edad relevante: para cada cohorte y cada grupo de edad los ponderadores suman la unidad. Los indicadores también fueron computados como el promedio simple entre los resultados de todos los países a fin de controlar por la incidencia de aquellos con mayor población.

En el gráfico 12 se observa el comportamiento de la participación laboral y la tasa de empleo para tres de las cohortes bajo análisis y siete intervalos de edad. Ellas 
CUADRO 3

Cohortes de nacimiento

(En intervalos por años de edad)

\begin{tabular}{|c|c|c|c|c|c|c|}
\hline Cohortes & $\begin{array}{c}\text { Inicios década } \\
1980\end{array}$ & $\begin{array}{c}\text { Finales década } \\
1980\end{array}$ & $\begin{array}{c}\text { Inicios década } \\
1990\end{array}$ & $\begin{array}{c}\text { Finales década } \\
1990\end{array}$ & $\begin{array}{c}\text { Inicios década } \\
2000\end{array}$ & $\begin{array}{c}\text { Finales década } \\
2000\end{array}$ \\
\hline 1960-1964 & $16-24$ & $21-29$ & $26-34$ & $31-39$ & $36-44$ & $41-49$ \\
\hline $1970-1974$ & & $15-19$ & $16-24$ & $21-29$ & $26-34$ & $31-39$ \\
\hline $1975-1979$ & & & $15-19$ & $16-24$ & $21-29$ & $26-34$ \\
\hline 1980-1984 & & & & $15-19$ & $16-24$ & $21-29$ \\
\hline 1985-1989 & & & & & $15-19$ & $16-24$ \\
\hline
\end{tabular}

Fuente: elaboración propia.

GRÁFICO 12

Tasas porcentuales de participación y de empleo

a través de tres cohortes de nacimiento

(Promedio ponderado)

A. Tasa de participación

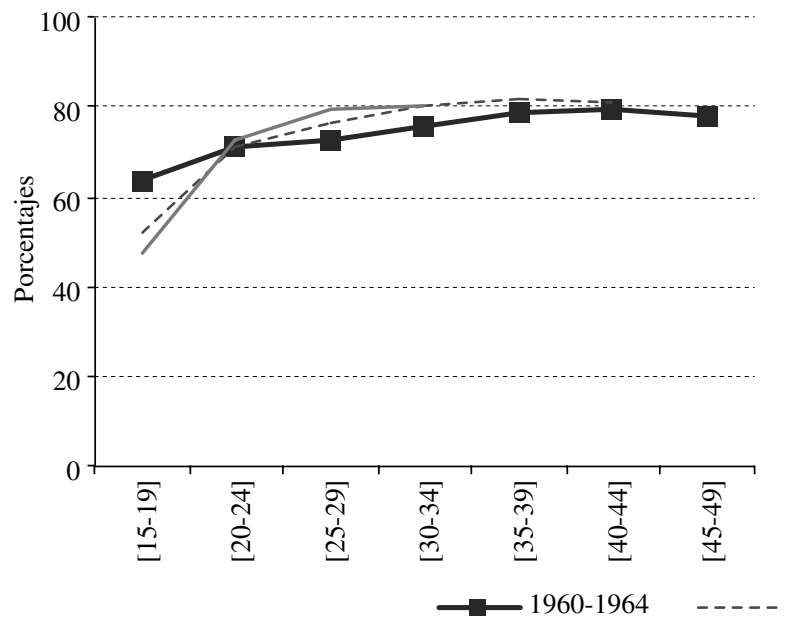

B. Tasa de empleo

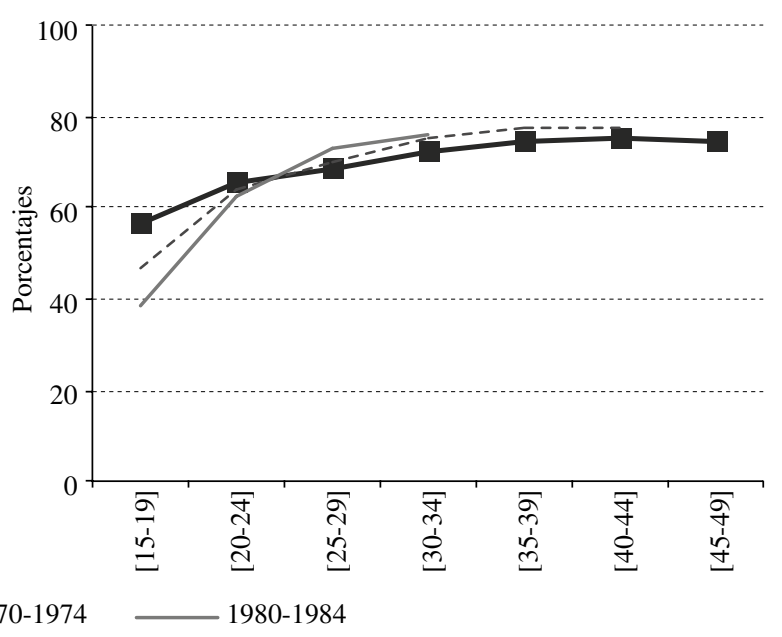

Fuente: elaboración propia sobre la Base de Datos Socioeconómicos para América Latina y el Caribe (SEDLAC) del Centro de Estudios Distributivos, Laborales y Sociales (CEDLAs) y el Banco Mundial.

Nota: $[\mathrm{x}, \mathrm{y}]$ intervalo que incluye ambos extremos.

son la cohorte más antigua (nacidos entre 1960 y 1964), una cohorte intermedia (nacidos entre 1970 y 1974) y una cohorte más joven (nacidos entre 1980 y 1984). Esto se realiza a modo de simplificación para poder advertir los patrones principales en el comportamiento de los indicadores laborales. Los resultados detallados para cada una de las cohortes se presentan en el cuadro A.8 del anexo.

Las diferencias entre cohortes para los primeros grupos de edad permiten advertir el patrón analizado en la sección III. Al considerar a jóvenes de cohortes más recientes, menguan las tasas de actividad y de empleo. Esto se advierte claramente respecto de los individuos que tienen entre 15 y 19 años de edad.
Con relación al grupo de edad siguiente, las tasas de participación y de empleo aumentan y la diferencia entre cohortes comienza a cerrarse. Los jóvenes de todas las cohortes ingresan en una trayectoria típica del ciclo de vida laboral: las tasas de participación y de empleo se incrementan con la edad, pero a un ritmo decreciente. La evidencia relevante que aporta este tipo de análisis es la reversión en la clasificación de cohortes una vez que los individuos se vuelven adultos. A partir de los 25 años, la participación y el empleo son superiores en los individuos de la cohorte más reciente con respecto a los adultos de cohortes anteriores. Esta misma trayectoria se observa al computar los resultados como un promedio simple. La distinción por género revela que respecto de 
los hombres la brecha entre cohortes comienza a cerrarse al llegar a la adultez y a partir de entonces no se advierte prácticamente ninguna diferencia. Entre las mujeres la recuperación en la tasa de actividad y de empleo es más rápida. A partir de los 20-24 años la cohorte más reciente supera a las anteriores y ese patrón se mantiene a lo largo de todo el ciclo de vida (véase el cuadro A.9 del anexo). La distinción por niveles educativos muestra que la recuperación de la cohorte más reciente es más lenta entre los individuos de nivel educativo superior (véase el cuadro A.10 del anexo).

La tasa de desempleo de los jóvenes de la cohorte más reciente sobrepasa ampliamente a la de los jóvenes de cohortes anteriores (véase el panel izquierdo del gráfico $13)^{6}$. A medida que los individuos continúan su carrera laboral, la tasa de desempleo se reduce. El desempleo de la cohorte del período de nacimiento 1970-1974 iguala al de la cohorte más antigua al alcanzar los 34-39 años, y a partir de entonces es inferior. La reducción en la tasa de desempleo de la cohorte del período de nacimiento 1980-1984 se produce a una mayor velocidad e iguala

${ }^{6}$ La discrepancia entre cohortes es aún más pronunciada al calcular el promedio simple, y refleja las diferencias de nivel entre países. el nivel de la cohorte de nacidos entre 1970 y 1974 al ingresar a la adultez. Este análisis revela que los individuos nacidos entre 1980 y 1984 tuvieron tasas de desempleo muy elevadas durante su juventud, pero su situación se recupera rápidamente durante la adultez. Trayectorias similares se advierten al distinguir entre hombres y mujeres, con una diferencia de nivel que favorece a los hombres. El análisis por niveles educativos deja ver patrones similares, con una mayor tasa de desempleo para los individuos de nivel educativo secundario $\mathrm{y}$ superior con respecto a los de nivel primario, lo que es compatible con los hallazgos de la sección III. En el nivel educativo primario las cohortes más recientes logran mejorar el desempeño de las generaciones anteriores, pero este comportamiento no se verifica en los niveles educativos secundario y superior al computar el promedio ponderado ${ }^{7}$.

El salario horario muestra una clara relación positiva con la edad en todas las cohortes de nacimiento (véase el panel derecho del gráfico 13). También se aprecia una mejora salarial al considerar cohortes más jóvenes,
${ }^{7}$ La mejora entre cohortes ocurre para todos los niveles educativos al calcular el promedio simple.

Tasas porcentuales de desempleo y de salario horario a través de tres cohortes de nacimiento (Promedio ponderado)

A. Tasa de desempleo

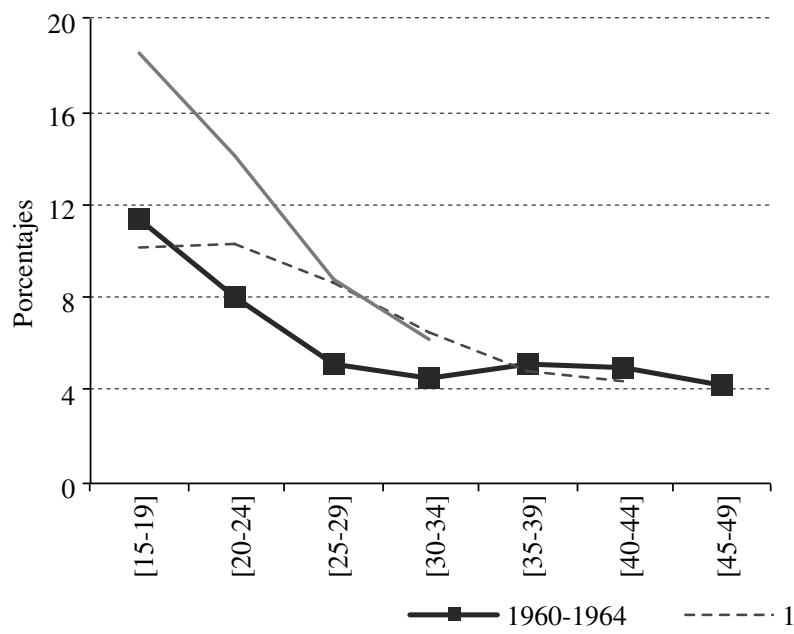

B. Salario horario

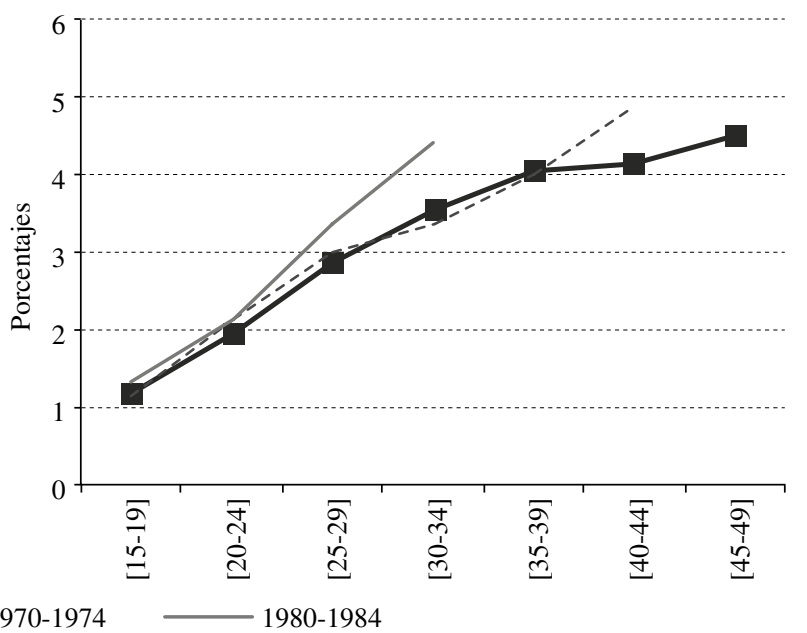

Fuente: elaboración propia sobre la Base de Datos Socioeconómicos para América Latina y el Caribe (SEDLAC) del Centro de Estudios Distributivos, Laborales y Sociales (CEDLAS) y el Banco Mundial.

Nota: $[\mathrm{x}, \mathrm{y}]$ intervalo que incluye ambos extremos. 
especialmente al comparar la cohorte de 1980-1984 con las anteriores. Esto es compatible con el análisis de la sección III, donde se vio que los salarios de jóvenes y adultos siguieron una trayectoria creciente en el tiempo. Nuevamente, las trayectorias no difieren entre hombres y mujeres, aunque el salario es más elevado para los primeros. Este patrón también aparece respecto de los individuos de nivel educativo primario, con un salario horario inferior en relación con la muestra completa. Entre los individuos de nivel secundario la recuperación se produce a un ritmo más lento y en el grupo de nivel superior la cohorte más reciente nunca iguala el desempeño de las cohortes anteriores.

El último indicador que se analiza en esta sección es la tasa de informalidad laboral. Del análisis del gráfico 14 surge: i) la informalidad laboral decrece con la edad hasta los primeros años de la adultez, cuando comienza a aumentar con el tiempo. En la sección III se mostró que la tasa de informalidad de los adultos siempre fue inferior a la de los jóvenes, pero el incremento en el tiempo fue más pronunciado para los primeros. La relación en forma de U que se advierte en el análisis de cohortes reflejaría ese fenómeno; ii) al considerar cohortes más recientes, la tasa de informalidad es superior en todas las etapas del ciclo de vida laboral; iii) la brecha comienza a cerrarse en la adultez para la cohorte más actual, que logra igualar el desempeño de la cohorte anterior, pero todavía está muy lejos de los niveles de la cohorte más antigua. Esto se cumple en relación con hombres y mujeres y podría estar indicando la existencia de algún tipo de penalidad vinculada a la condición de informalidad durante la juventud; iv) los resultados por niveles educativos muestran que en el nivel superior la cohorte más actual reduce rápidamente su tasa de informalidad, aproximándose a la observada en la cohorte de 1970-1974, entre los 30 y 34 años. En los niveles educativos primario y secundario la cohorte más reciente reduce su tasa de informalidad al acumular experiencia, pero no logra cerrar la brecha con la cohorte de 1970-1974. La penalidad vinculada a la condición de informalidad en la juventud sería más drástica en estos casos.

GRÁFICO 14

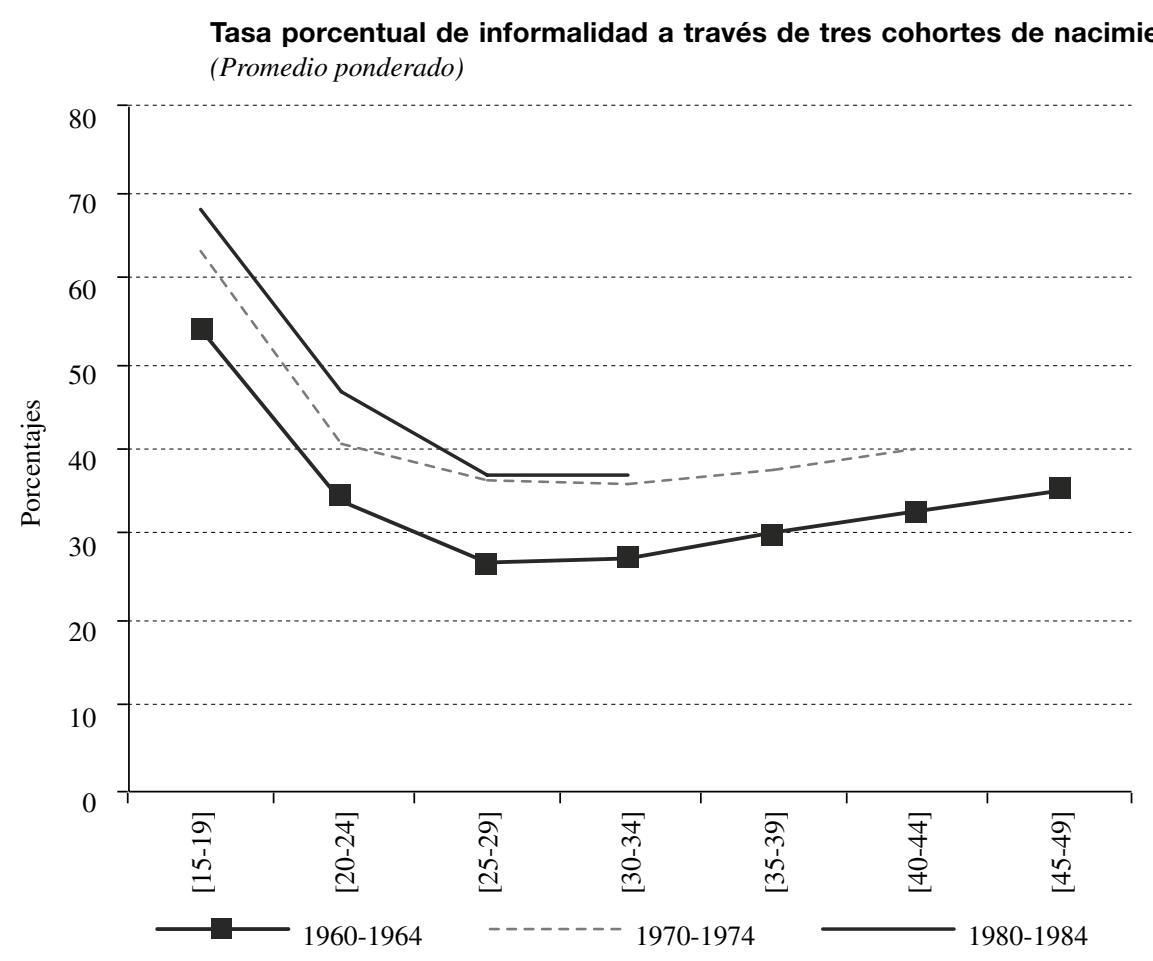

Fuente: elaboración propia sobre la Base de Datos Socioeconómicos para América Latina y el Caribe (sedLaC) del Centro de Estudios Distributivos, Laborales y Sociales (CEDLAS) y el Banco Mundial.

Nota: $[\mathrm{x}, \mathrm{y}]$ intervalo que incluye ambos extremos. 


\section{V}

\section{Conclusiones}

En este trabajo se han destacado distintas características y dinámicas de los mercados laborales de América Latina en las últimas tres décadas. En primer lugar, el análisis de la evolución de indicadores laborales reveló que la situación de los jóvenes se ha deteriorado en el transcurso del tiempo con una mejora hacia finales de la década de 2000. El período de transición entre la etapa de formación y el ingreso al mercado de trabajo se ha prolongado debido a la extensión de la etapa de acumulación de capital humano, pero la situación de los jóvenes que permanecieron en el mercado laboral se ha debilitado. A pesar de esta mejora en el nivel de calificación, la tasa de desempleo aumentó al igual que la tasa de informalidad, denotando el surgimiento o endurecimiento de barreras a la entrada del sector formal, que son particularmente difíciles de sortear para los jóvenes de bajo nivel educativo. Esto sugiere que los esfuerzos realizados para mejorar la situación de los jóvenes en el mercado de trabajo deben mantenerse para prolongar la recuperación.

En segundo lugar, los jóvenes que enfrentan perspectivas desfavorables al entrar por primera vez al mercado de trabajo logran ingresar en una trayectoria típica del ciclo de vida laboral a medida que acumulan experiencia. Esto permite que los jóvenes pertenecientes a cohortes de nacimiento más actuales logren en la adultez mayores tasas de empleo, menores tasas de desempleo y un mejor salario horario que los adultos pertenecientes a cohortes anteriores. En el caso de la informalidad laboral, su nivel decrece al pasar de la juventud a la adultez, pero las generaciones más recientes no logran igualar el desempeño de generaciones pasadas, lo que indica la existencia de algún tipo de penalidad relacionada con la condición de informalidad en la juventud. Esto ocurre principalmente entre los individuos de nivel educativo primario. Aun cuando el empleo en el sector informal puede cumplir el papel de entrenamiento laboral y acumulación de experiencia (Bosch y Maloney, 2010; Cunningham y Bustos, 2011), este análisis muestra que la experiencia en el sector informal durante la juventud puede no ser suficiente para generar la movilidad hacia empleos protegidos en la adultez (Cruces, Ham y Viollaz, 2012).

El análisis desde una perspectiva de género reveló que las mujeres jóvenes se han incorporado al mercado de trabajo, pero la proporción de las que no estudian y tampoco trabajan sigue sobrepasando con creces el valor registrado por los hombres; y entre aquellas que participan activamente en el mercado de trabajo, su tasa de desempleo también supera el valor obtenido por ellos. Las mejores perspectivas laborales de los jóvenes hacia fines de la década de 2000 repercutieron fuertemente en algunos casos entre las mujeres: la mejora salarial fue más marcada para ellas, al igual que la reducción en la informalidad laboral.

En resumen, estos resultados indican que las perspectivas desfavorables que caracterizaron la inserción laboral de la población joven durante la década de 1990 e inicios de la de 2000 comenzaron a revertirse en los últimos años de esta última década. Además de esto, el análisis de ciclo de vida mostró que los jóvenes mejoran su desempeño en el mercado de trabajo una vez que se hacen adultos. Si bien este resultado es muy alentador, es importante tener en cuenta que la recuperación toma tiempo, y las características de la inserción juvenil pueden traducirse en penalidades durante la adultez en términos de menores salarios, mayores posibilidades de desempleo o de trabajo informal.

Al considerar que el debilitamiento de la situación laboral de los jóvenes hasta inicios de los años 2000 ocurrió a pesar de su mayor nivel educativo, los resultados conducen al sostenimiento de políticas orientadas a asistirlos en la obtención de su primer empleo. La elevada tasa de informalidad en este grupo y la evidencia reciente de que un empleo informal durante la juventud no asegura el entrenamiento suficiente para moverse al sector formal (Cruces, Ham y Viollaz, 2012), agrega otra dificultad al diseño de políticas. Medidas como incentivos monetarios a la contratación de trabajadores jóvenes o un menor nivel de salario mínimo en este rango etario podrían corregir una situación en que el salario es alto desde el punto de vista del empleador, generando incentivos adicionales al registro de trabajadores jóvenes. Otro tipo de estrategias pueden intentar reducir los costos de búsqueda laboral, por ejemplo, resolviendo el desajuste entre el lugar en que vive el desempleado y aquel donde se encuentran los empleos formales mediante subsidios al transporte.

La elevada tasa de desempleo de la población juvenil junto con un nivel de educación creciente también conduce a plantear un interrogante sobre la calidad del sistema educativo en la región. América Latina ha mostrado avances significativos en las últimas décadas 
en términos de acceso, pero también se ha detectado una mayor segmentación en resultados educativos y calidad de la oferta (CEPAL, 2011). En un contexto de acceso desigual a las oportunidades educativas es posible esperar que las inequidades sociales persistan e incluso se amplíen en la transición desde el sistema educativo al mercado laboral. Aquí se abre un espacio para la acción en el plano educativo, mediante medidas orientadas a ampliar y fortalecer la enseñanza en los niveles educativos iniciales y garantizar la formación para el trabajo como una manera de suavizar la transición al mundo laboral asegurando la adaptación a los cambios productivos y tecnológicos.

Otra estrategia de empleo consiste en facilitar el crecimiento de emprendimientos o actividades productivas propias. La disminución de las limitaciones que impiden que los desempleados desarrollen una actividad propia representaría un apoyo a las iniciativas de los jóvenes, por ejemplo, mediante la eliminación de barreras legales y restricciones crediticias.

Por último, es relevante que en toda política que se implemente se consideren las diferencias en la inserción y condiciones de empleo de hombres y mujeres y se eliminen aquellas que son una manifestación del fenómeno de discriminación en el mercado de trabajo.

Como conclusión, la mejora en la calidad de la primera experiencia juvenil en el mercado de trabajo debe ser prioritaria. Aun cuando la creciente inestabilidad del mercado laboral atenta contra la necesidad de cierta permanencia en el empleo de los jóvenes (Weller, 2006), las medidas de políticas que logren afianzar la posición de estos, en términos de estabilidad y calidad de sus empleos, tendrán efectos duraderos en el ciclo de vida de los individuos. 
ANEXO

CUADRO A.1

\section{Encuestas de hogares}

\begin{tabular}{|c|c|c|}
\hline Argentina & $\begin{array}{l}1980-2002 \\
2003-2012\end{array}$ & $\begin{array}{l}\text { Encuesta Permanente de Hogares - Puntual } \\
\text { Encuesta Permanente de Hogares - Continua }\end{array}$ \\
\hline Brasil & $1981-2011$ & Pesquisa Nacional por Amostra de Domicilios \\
\hline Chile & $1987-2011$ & Encuesta de Caracterización Socioeconómica Nacional \\
\hline Costa Rica & $\begin{array}{l}1989-2009 \\
2010\end{array}$ & $\begin{array}{l}\text { Encuesta de Hogares de Propósitos Múltiples } \\
\text { Encuesta Nacional de Hogares }\end{array}$ \\
\hline El Salvador & $1991-2010$ & Encuesta de Hogares de Propósitos Múltiples \\
\hline Honduras & $1990-2011$ & Encuesta Permanente de Hogares de Propósitos Múltiples \\
\hline México & $1989-2010$ & Encuesta Nacional de Ingresos y Gastos de los Hogares \\
\hline Panamá & $\begin{array}{l}1989-1991 \\
1995-2012\end{array}$ & $\begin{array}{l}\text { Encuesta de Hogares, Mano de Obra } \\
\text { Encuesta de Hogares }\end{array}$ \\
\hline Uruguay & $1989-2011$ & Encuesta Continua de Hogares \\
\hline Venezuela (República Bolivariana de) & $1989-2011$ & Encuesta de Hogares por Muestreo \\
\hline
\end{tabular}

Fuente: elaboración propia sobre la Base de Datos Socioeconómicos para América Latina y el Caribe (SEDLAC) del Centro de Estudios Distributivos, Laborales y Sociales (CEDLAs) y el Banco Mundial.

CUADRO A. 2

Años utilizados para construir ponderadores

(Inicios década 1980 - finales década 2000)

\begin{tabular}{|c|c|c|c|c|c|c|c|c|c|c|}
\hline Período & Argentina & Brasil & Chile & Costa Rica & El Salvador & Honduras & México & Panamá & Uruguay & $\begin{array}{c}\text { Venezuela } \\
\text { (República } \\
\text { Bolivariana de) }\end{array}$ \\
\hline Inicios década 1980 & 1980 & 1981 & $\ldots$ & $\ldots$ & $\ldots$ & $\ldots$ & $\ldots$ & $\ldots$ & $\ldots$ & $\ldots$ \\
\hline Finales década 1980 & 1989 & 1989 & 1987 & 1989 & $\ldots$ & $\ldots$ & 1989 & 1989 & 1989 & 1989 \\
\hline Inicios década 1990 & 1992 & 1992 & 1992 & 1992 & 1991 & 1992 & 1992 & 1991 & 1992 & 1992 \\
\hline Finales década 1990 & 1998 & 1998 & 1998 & 1998 & 1998 & 1998 & 1998 & 1998 & 1998 & 1998 \\
\hline Inicios década 2000 & 2003 & 2003 & 2003 & 2003 & 2003 & 2003 & 2002 & 2003 & 2003 & 2003 \\
\hline Finales década 2000 & 2009 & 2009 & 2009 & 2009 & 2009 & 2009 & 2008 & 2009 & 2009 & 2009 \\
\hline
\end{tabular}

Fuente: elaboración propia sobre la Base de Datos Socioeconómicos para América Latina y el Caribe (SEDLAC) del Centro de Estudios Distributivos, Laborales y Sociales (CEDLAs) y el Banco Mundial. 


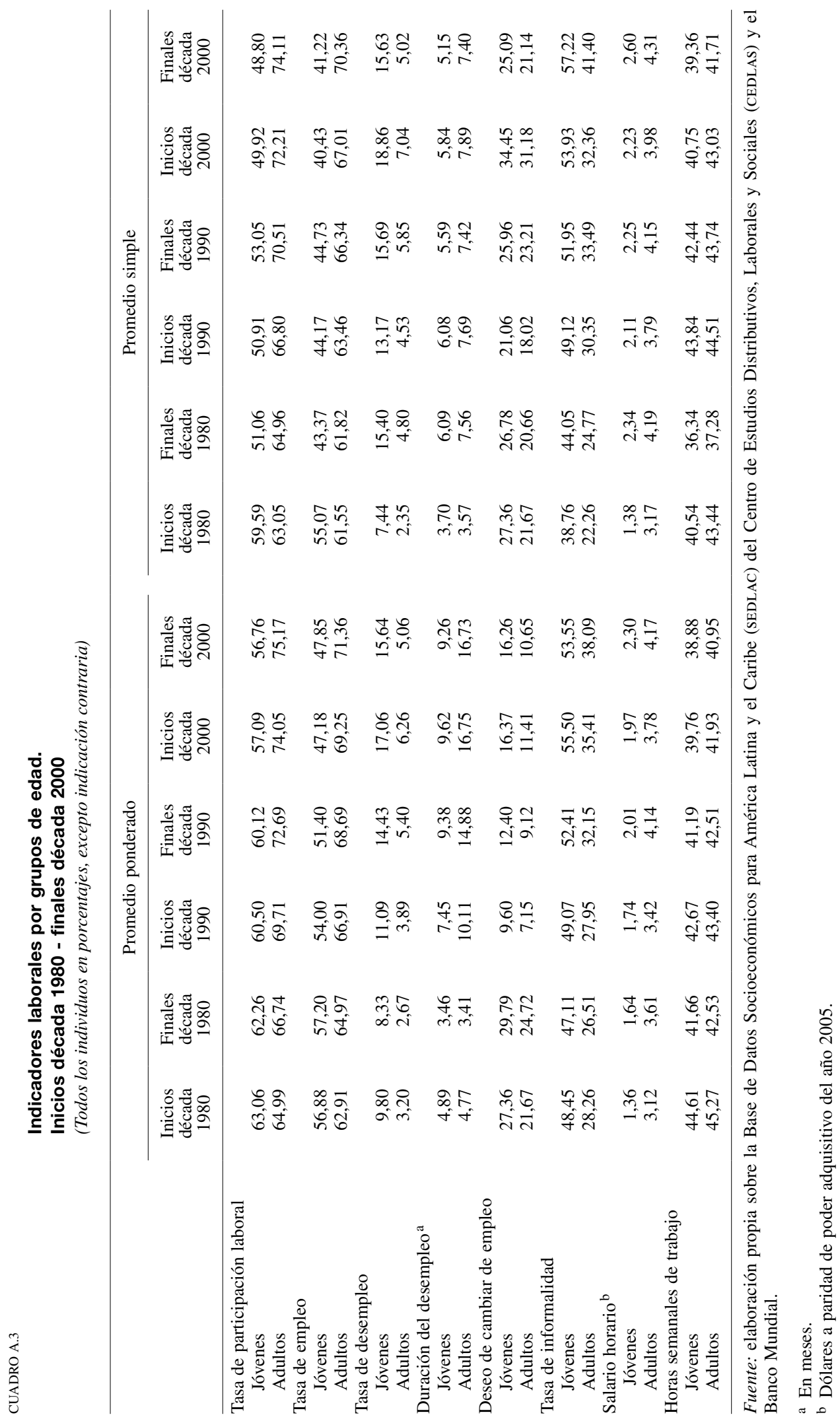




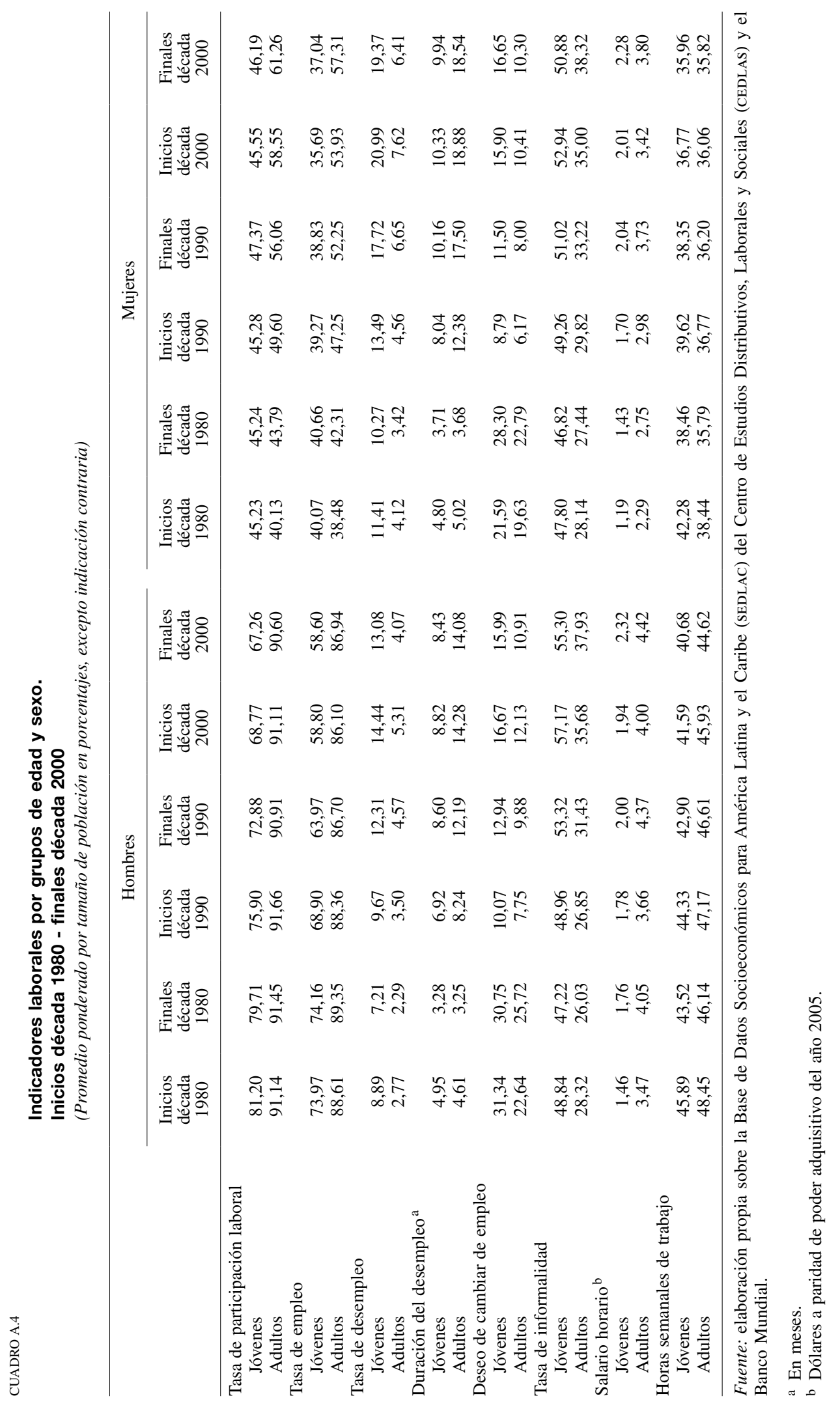




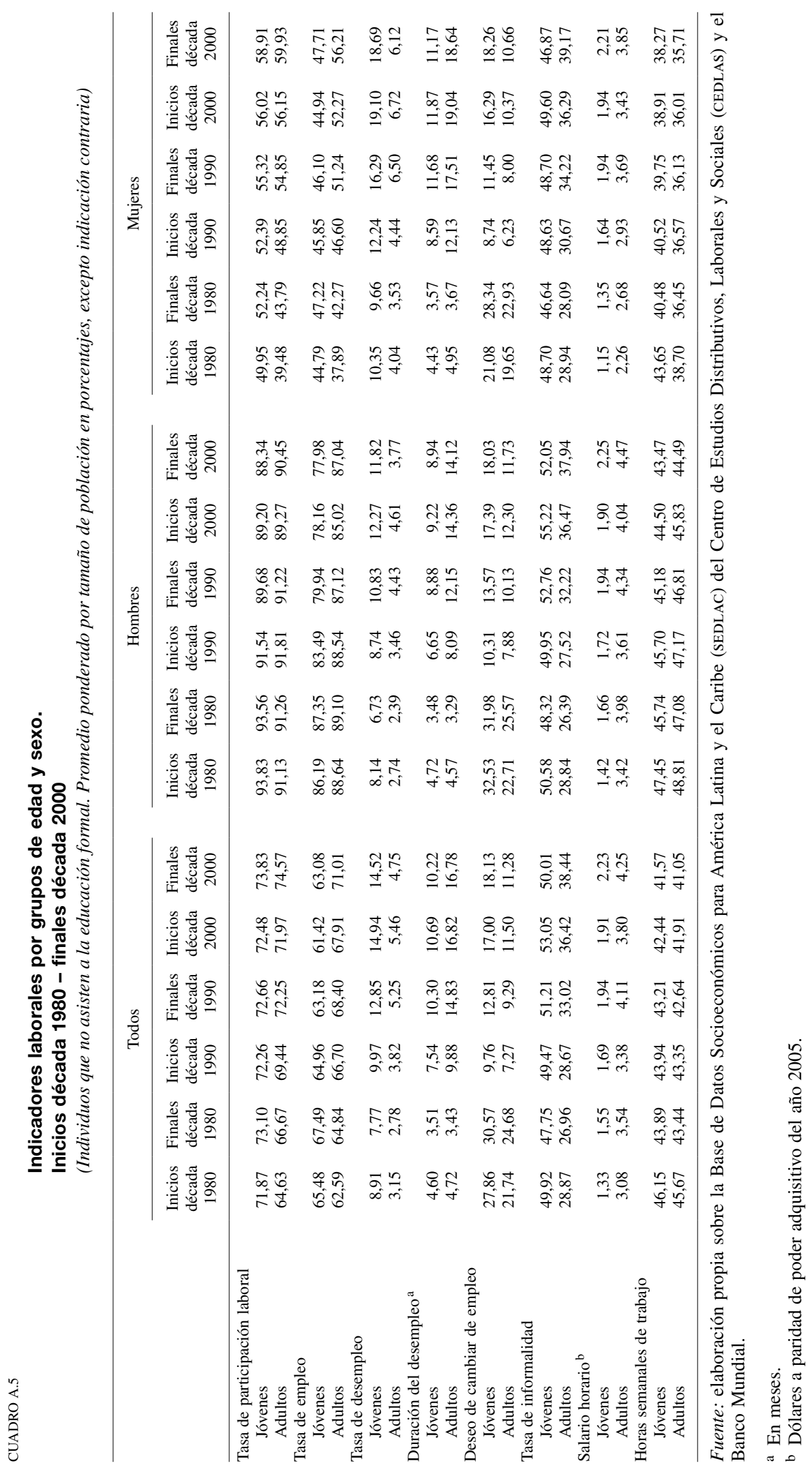




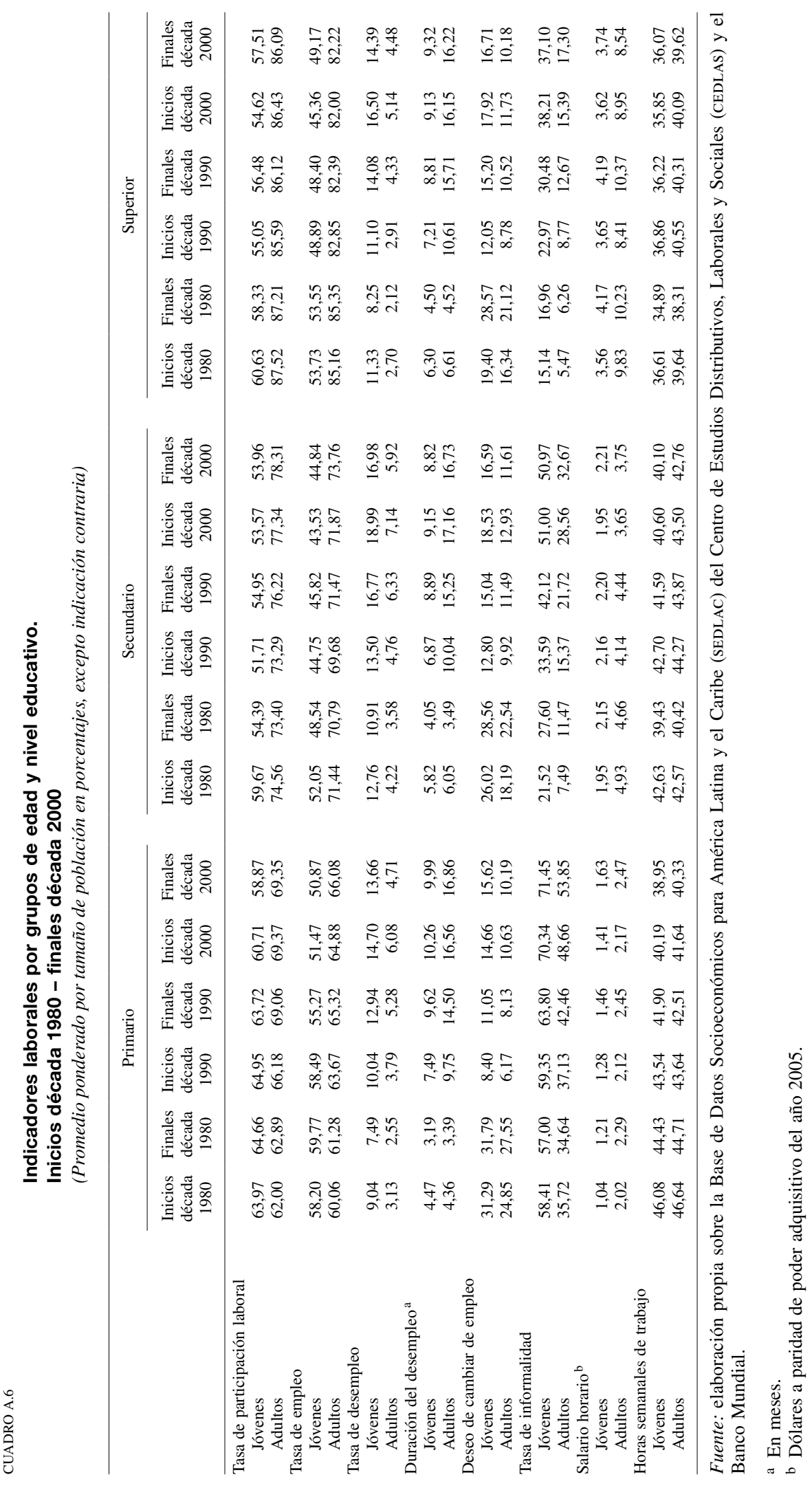




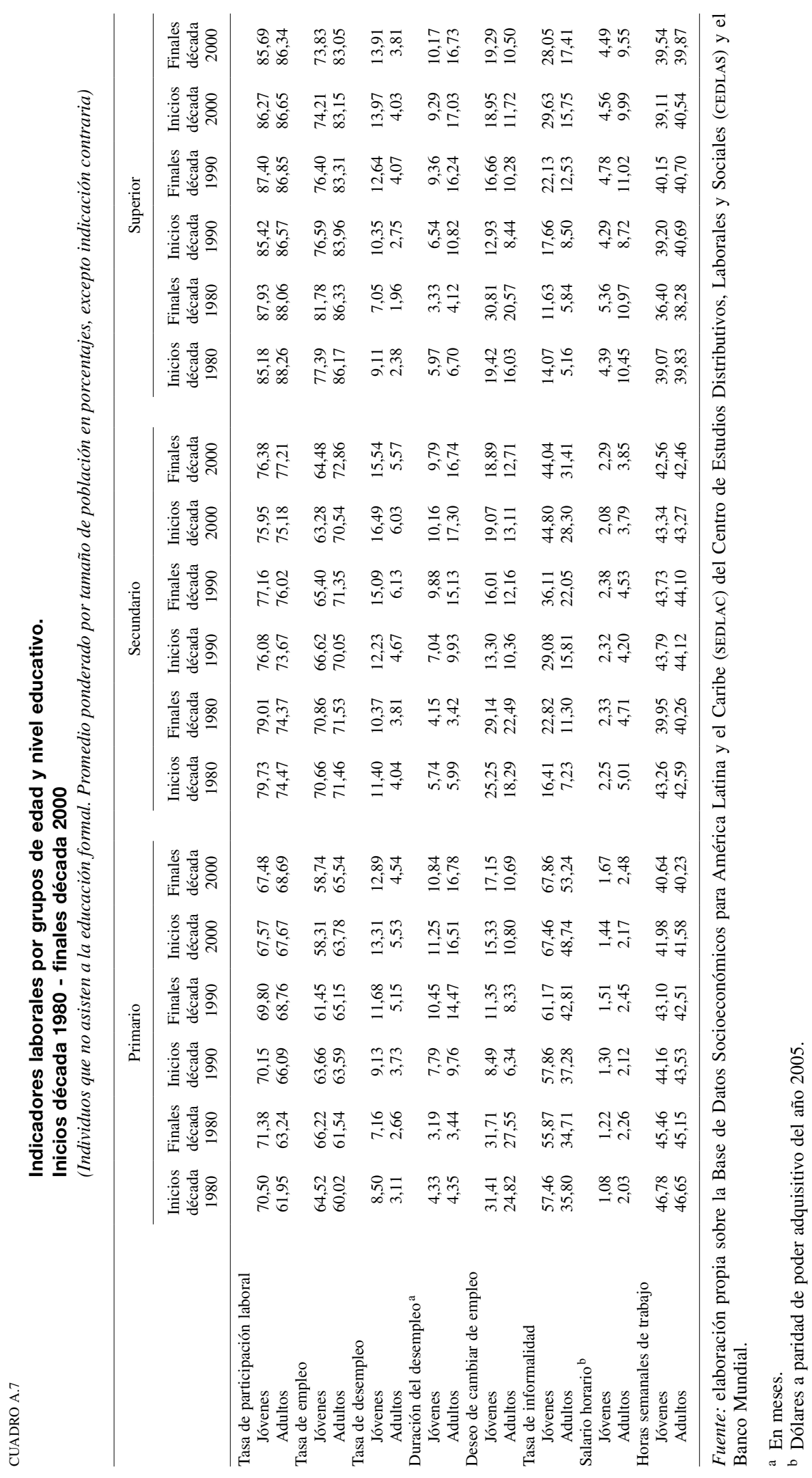




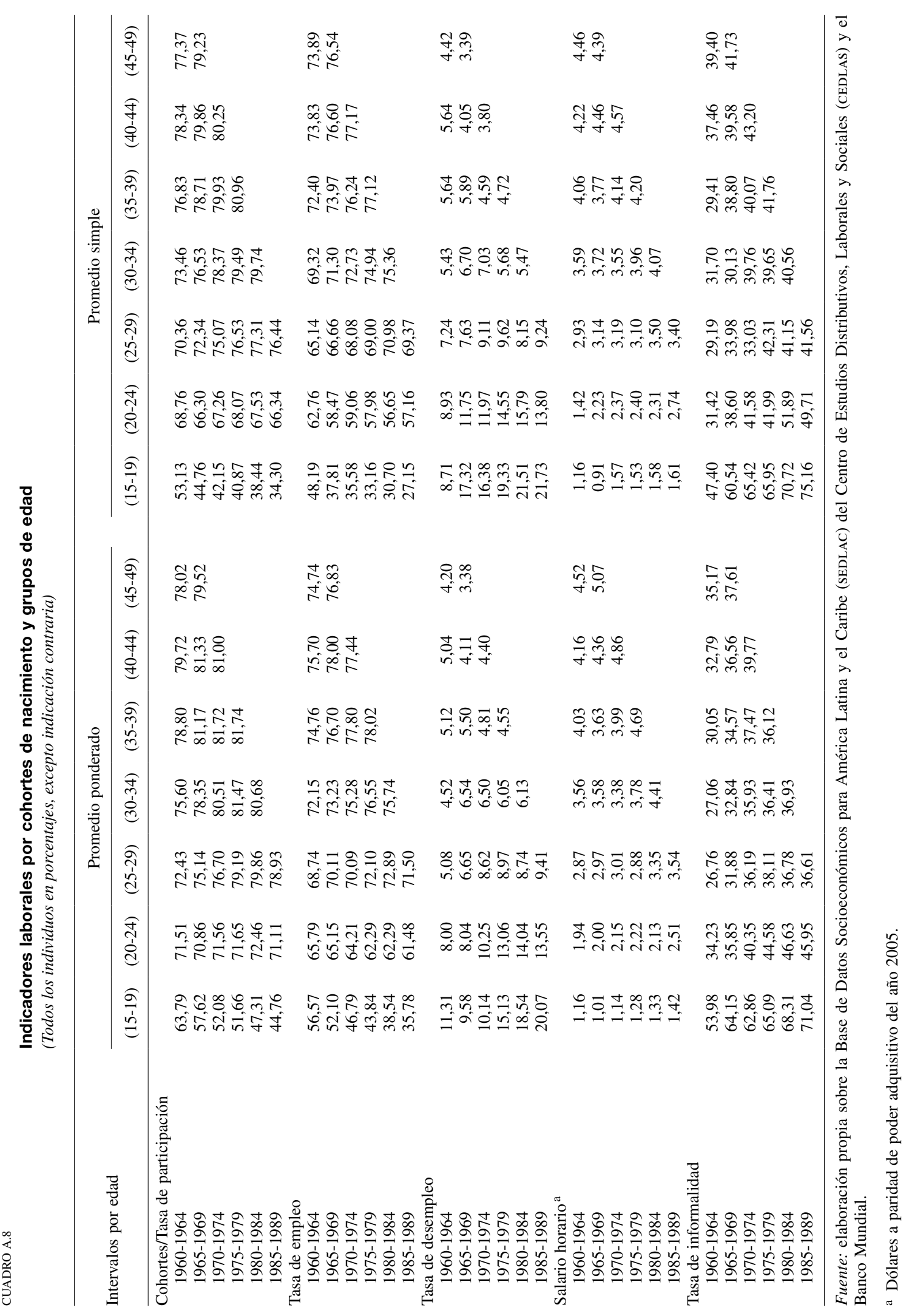




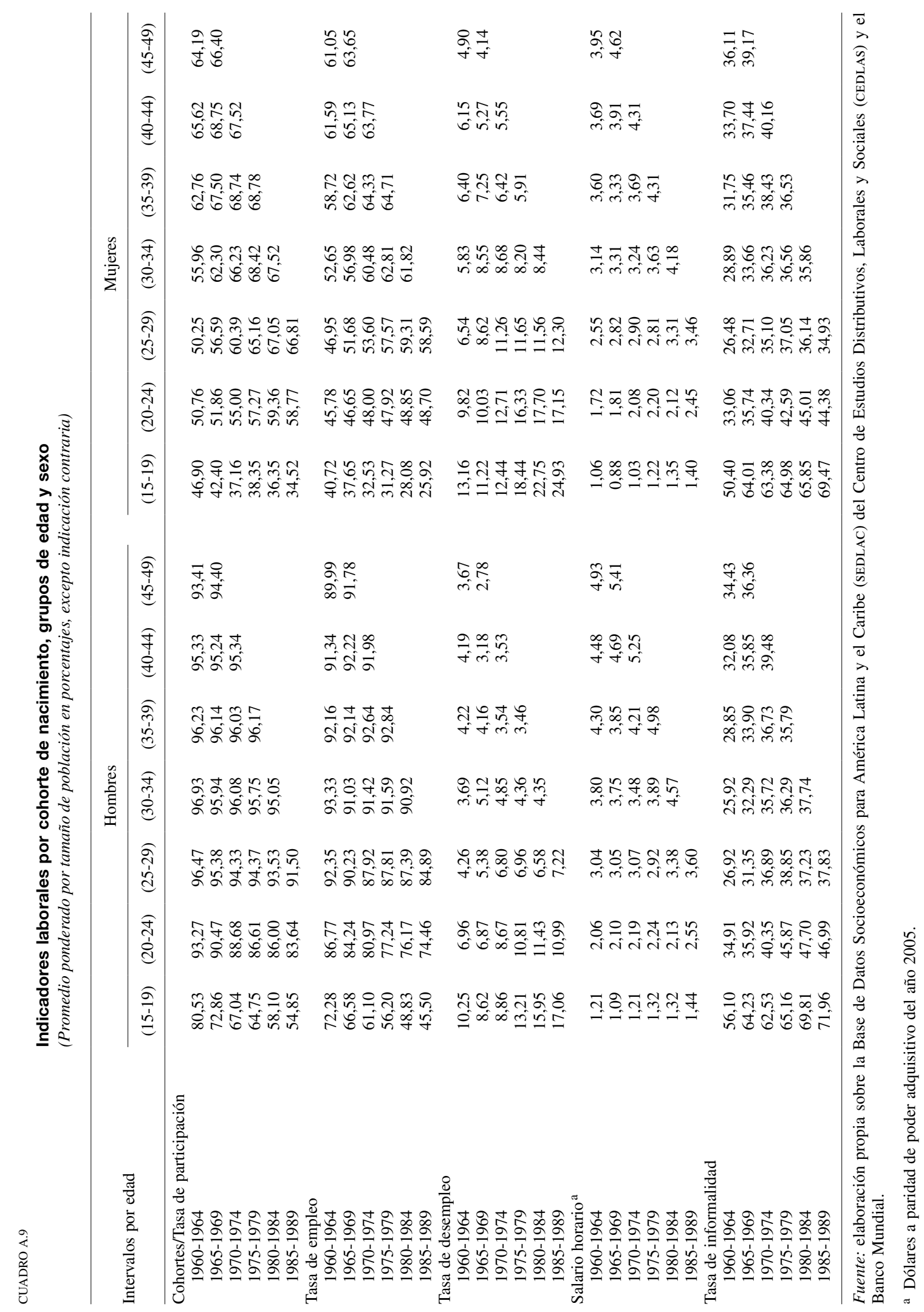




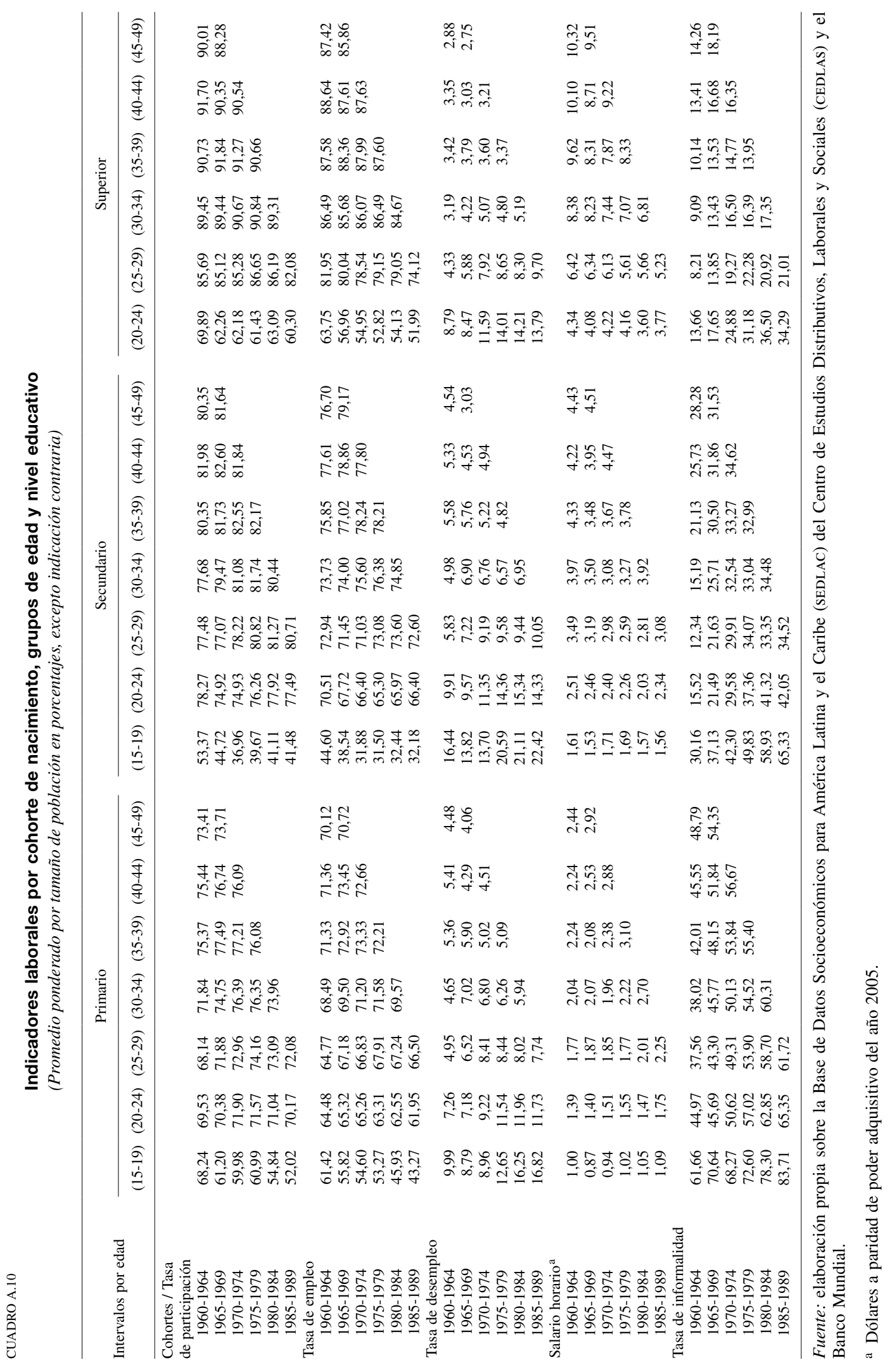




\section{Bibliografía}

Bassi, M. y S. Galiani (2009), "Youth Labor Market in Latin America: A Descriptive Analysis", Washington, D.C., Banco Inter-Americano de Desarrollo, inédito.

Banco Mundial (2012), World Development Indicators, Washington, D.C.

Bell, D. y D. Blanchflower (2010), "Youth unemployment: déjà vu?", IZA Discussion Paper, $\mathrm{N}^{\circ} 4705$, Bonn, Institute for the Study of Labor, enero.

Blanchflower, D. y R.B. Freeman (2000), "The declining economic status of young workers in OECD countries", Youth Employment and Joblessness in Advanced Countries, D.G. Blanchflower y R.B. Freeman (eds.), Cambridge, Massachusetts, National Bureau of Economic Research.

Bosch, M. y W. Maloney (2010), "Comparative analysis of labor market dynamics using Markov processes: an application to informality", Labour Economics, vol. 17, N 4, Amsterdam, Elsevier.

Breen, R. (1992), "Job changing and job loss in the Irish youth labor market: a test of a general model", European Sociological Review, vol. 8, $\mathrm{N}^{\circ} 2$, Oxford, Oxford University Press.

Cárdenas, M., R. de Hoyos y M. Székely (2011), The Idle Youth in Latin America: A Persistent Problem in a Decade of Prosperity, Washington, D.C., The Brookings Institution.

CEPAL (Comisión Económica para América Latina y el Caribe) (2011), Panorama Social de América Latina 2010 (LC/G.2481-P), Santiago de Chile. Publicación de las Naciones Unidas, $\mathrm{N}^{\circ}$ de venta: S.11.II.G.6.

CePalstat (Bases de Datos y Publicaciones Estadísticas) (2012), julio [en línea] http://www.eclac.org/estadisticas.

Cruces, G., A. Ham y M. Viollaz (2012), "Scarring Effects of Youth Unemployment and Informality: Evidence from Brazil", inédito.

Cunningham, W. y J. Bustos (2011), "Youth employment transitions in Latin America", Policy Research Working Papers, $\mathrm{N}^{\circ}$ 5521, Washington, D.C., Banco Mundial.
Dolado, J., F. Felgueroso y J. Jimeno (2000), "Explaining youth labor market problems in Spain: crowding-out, institutions, or technology shifts?", IZA Discussion Paper, $\mathrm{N}^{\circ} 142$, Bonn, Institute for the Study of Labor, febrero.

Fawcett, C. (2001), "Los jóvenes latinoamericanos en transición: Un análisis sobre el desempleo juvenil en América Latina y el Caribe", Serie Documentos de Trabajo Mercado Laboral, Washington, D.C., Banco Interamericano de Desarrollo.

Marchionni, M., G. Bet y A. Pacheco (2007), "Empleo, educación y entorno social de los jóvenes: Una nueva fuente de información", Documento de Trabajo, $\mathrm{N}^{\circ}$ 61, Centro de Estudios Distributivos, Laborales y Sociales (CEDLAS).

Maurizio, R. (2011), "Trayectorias laborales de los jóvenes en Argentina: ¿Dificultades en el mercado de trabajo o carrera laboral ascendente?", serie Macroeconomía del Desarrollo, $\mathrm{N}^{\circ} 109$ (LC/L.3302-P), Santiago de Chile, Comisión Económica para América Latina y el Caribe (CEPAL). Publicación de las Naciones Unidas, $\mathrm{N}^{\circ}$ de venta: S.11.II.G.24. (2010), "Enfoque de género en las instituciones laborales y las políticas del mercado de trabajo en América Latina", serie Macroeconomía del Desarrollo, № 104 (LC/L.3230-P), Santiago de Chile, Comisión Económica para América Latina y el Caribe (CEPAL). Publicación de las Naciones Unidas, $\mathrm{N}^{\circ}$ de venta: S.10.II.G.34

Neumark, D. (2002), "Youth labor markets in the United States: shopping around vs. staying put", The Review of Economics and Statistics, vol. 84, $\mathrm{N}^{\circ} 3$, Cambridge, Massachusetts, The MIT Press.

Neumark, D. y O. Nizalova (2007), "Minimum wage effects in the longer run", Journal of Human Resources, vol. 46, $\mathrm{N}^{\circ} 2$, Madison, University of Wisconsin Press.

SEDLAC (Base de Datos Socioeconómicos para América Latina y el Caribe) (2013) [en línea] http://sedlac.econo.unlp.edu.ar.

Weller, J. (ed.) (2006), Los jóvenes y el empleo en América Latina. Desafios y perspectivas ante el nuevo escenario laboral, Santiago de Chile, Comisión Económica para América Latina y el Caribe (CEPAL). 